\title{
On Normal Extensions of Unbounded Operators. III. Spectral Properties
}

\author{
By \\ Jan StocheL* and Franciszek H. SzafranieC*
}

\section{Introduction}

1. This is the last part of our trilogy devoted to systematic exposition of the fundamentals of the theory of unbounded subnormal operators. In the previous papers [17 and 18] we focus our interest on conditions under which normal extensions exist. The present part concerns spectral properties of subnormal operators as related to those of their normal extensions.

In our approach we have been trying to unify features of symmetric operators, the classical object of the theory, from one side, and bounded subnormal operators, from the other. The most spectacular representative of this fusion is the creation operator

$$
x-\frac{d}{d x}
$$

It is apparently a differential operator but also it has an analytic model. Existence of such a model is one of the major topics of this paper. On the way to achieving this goal we consider the following questions: minimality of normal extensions, their uniqueness and different kinds of spectral relations.

In particular, we discuss two sorts of minimality: of spectral type and of cyclic type. For unbounded operators these two notions need not coincide (for bounded they always do). This impacts the uniqueness question.

However, in spite of the lack of uniqueness, basic spectral relations can be carried over from the bounded case to the unbounded one. In particular, the spectral inclusion property holds true (we have taken the opportunity to collect here all possible spectral relations).

Among bounded subnormal operators there are those which have analytic models in Hardy-like spaces (cf. [3]). Unfortunately, the spectrum of an unbounded subnormal operator may have no boundary; this means that Bargmannlike models [4] are preferable. Within the class of cyclic unbounded subnormal operator we are able to work out analytic models of both types, understanding cyclicity of an operator in the polynomial sense (though some of our results

Communicated by H. Araki, August 8, 1988.

* Instytut Matematyki, Uniwersytet Jagielloński, 30-059 Kraków, Reymonta 4, Poland. 
are still true for rationally cyclic operators, as in the bounded case [8]).

The paper ends in considering operators which behave like subnormal weighted shifts.

Some conventions. We use the asterisk * for conjugation of complex numbers as well as for taking adjoints of operators, while the dash - we reserve for closure operations. As usual, $\boldsymbol{C}[z]$ resp. $\boldsymbol{C}\left[z, z^{*}\right]$ stands for the polynomials (of complex coefficients) in $z$ resp. in $z$ and $z^{*}$ (sometimes we will call members of $\boldsymbol{C}[z]$ analytic polynomials). All the operators we consider in this paper are supposed to be densely defined.

\section{Minimality of Spectral Type}

2. Let $S$ be a densely defined linear operator in a complex Hilbert space $\mathscr{H}$. $S$ is said to be subnormal if there exists another Hilbert space $\mathcal{K}$ containing ${ }^{1}$ $\mathscr{H}$ and a densely defined normal operator $N$ in $\mathcal{K}$ such that

$$
\mathscr{D}(S) \subset \mathscr{D}(N) \cap \mathscr{H} \quad \text { and } \quad S f=N f, f \in \mathscr{D}(S) .
$$

One of the questions we would like to consider here is, in analogy with the bounded case, minimality of the extension $N$. Unlike the bounded case we have several, in general non-equivalent, ways of understanding minimality. In the bounded case all these notions coincide and minimality always forces uniqueness of the normal extension up to unitary isomorphism.

The most general definition of minimality seems to be the following one: a normal extension $N$ of $S$ is said to be minimal of spectral type if the only closed subspace of $\mathcal{K}$ reducing $N$ and containing $\mathcal{K}$ is $\mathcal{K}$ itself (recall that a closed subspace $\mathscr{M}$ of $\mathcal{K}$ reduces an unbounded closed operator, say $N$, if $P N \subset N P$ where $P$ is the orthogonal projection of $\mathcal{K}$ onto $\mathscr{M}$ ).

Two normal extensions $N_{1}$ and $N_{2}$ of $S$ acting in Hilbert spaces $\mathcal{K}_{1}$ and $\mathcal{K}_{2}$ respectively, are said to be $\mathscr{K}$-equivalent if there exists a unitary operator $U: \mathcal{K}_{1} \rightarrow \mathcal{K}_{2}$ such that

$$
U f=f, f \in \mathscr{H} \quad \text { and } \quad U N_{1}=N_{2} U \text {. }
$$

Dropping the first of these two conditions we get that $N_{1}$ and $N_{2}$ are (unitarily) equivalent in the usual sense.

Minimal normal extensions of spectral type always exist. To make this evident we need some notations. Let $E$ be the spectral measure of a normal operator $N$ acting in a Hilbert space $\mathcal{K}$. If $\mathcal{H}$ is a closed linear subspace of $\mathcal{K}$, then we denote by $\mathscr{K}_{s}[N]$ the closed linear span of $\{E(\sigma) f: f \in \mathcal{K}, \sigma$ a Borel subset of $\boldsymbol{C}\}$. The space $\mathscr{H}_{s}[N]$ reduces the spectral measure $E$ of $N$ and consequently $\mathscr{K}_{s}[N]$ does $N$ too. Denote by $N_{\mathscr{H}}$ the operator acting in

1 "Containing" ought to be read as "containing an isometric image of"; this is just what occurs in mathematical reality. 
$\mathscr{H}_{s}[N]$ and defined by

$$
\mathscr{D}\left(N_{\mathscr{H}}\right)=\mathscr{D}(N) \cap \mathscr{H}_{s}[N], \quad N_{\mathscr{H}} f=N f, \quad f \in \mathscr{D}\left(N_{\mathscr{H}}\right) .
$$

Proposition 1. Let $S$ be a subnormal operator in $\mathcal{H}$ and $N$ its normal extension in $\mathcal{K}$. Then

$(\alpha) \quad N_{\mathscr{A}}$ is a minimal normal extension of spectral type of $S$,

( $\beta$ ) $N$ is a minimal normal extension of spectral type of $S$ if and only if $\mathcal{K}=$ $\mathscr{H}_{s}[N]$,

$(\gamma)$ if $N_{1}$ and $N_{2}$ are two normal extensions of $S$ which are $\mathcal{H}$-equivalent and $N_{1}$ is minimal of spectral type, then so is $N_{2}$,

$(\delta)$ if $N$ is a minimal normal extension of spectral type of $S$, which acts in $\mathcal{K}$, then $\operatorname{dim} \mathcal{K}=\operatorname{dim} \mathscr{H}^{2}$. Consequently the spaces two minimal extensions of spectral type of $S$ act in are unitarily isomorphic.

Proof. The proof of $(\alpha)$ and $(\beta)$ follows easily from the observation that $\mathcal{H}_{s}[N]$ reduces the spectral measure $E$ of the normal extension $N$ and consequently the operator $N$ itself. The part $(\gamma)$ of the conclusion follows straightforwardly.

Now we proceed to the proof of $(\delta)$. If $\operatorname{dim} \mathscr{H}$ is finite, then $S$ is normal itself. Suppose $\operatorname{dim} \mathscr{H} \geqq \aleph_{0}$. Let $\mathcal{E}$ be an orthonormal basis of $\mathscr{H}$. Let $\mathfrak{B}$ be a countable algebra generating the $\sigma$-algebra of all Borel sets on $\boldsymbol{C}$. The classical Carathéodory extension theorem [5, p. 19] permits us to find for each vector $f \in \mathscr{H}$ and each Borel set $\sigma$ a sequence $\left\{\sigma_{n}\right\} \subset \mathfrak{B}$ such that $\left\langle E\left(\left(\sigma \backslash \sigma_{n}\right) \cup\right.\right.$ $\left.\left(\sigma_{n} \backslash \sigma\right)\right) f, f>\rightarrow 0$ as $n \rightarrow \infty$, where $E$ is the spectral measure of $N$. This implies that $E\left(\sigma_{n}\right) f \rightarrow E(\sigma) f$. Since $\mathcal{K}=\mathscr{H}_{s}[N]$ we get $\mathcal{K}=\operatorname{clolin}\{E(\sigma) e: e \in \mathcal{E}, \sigma \in \mathfrak{B}\}$. This implies that $\operatorname{dim} \mathscr{K} \leqq \operatorname{card}\{E(\sigma) e: e \in \mathcal{E}, \sigma \in \mathfrak{B}\}$ and, since $\mathfrak{B}$ is countable, $\operatorname{card}\{E(\sigma) e: e \in \mathcal{E}, \sigma \in \mathfrak{B}\}=\operatorname{card} \mathcal{E}=\operatorname{dim} \mathscr{H}$. Thus $\operatorname{dim} \mathscr{K}=\operatorname{dim} \mathcal{K}$.

The equivalence $(\beta)$ justifies the name we have chosen for this kind of minimality.

3. Unfortunately, minimality of spectral type need not entail uniqueness. More precisely, it may happen (cf. Example 1 below) that two minimal normal extensions of spectral type are not equivalent at all, though the spaces they act in are, by condition $(\delta)$ of Proposition 1 , isomorphic.

In spite of this diversity the basic spectral inclusion property (i. e. conclusion $9^{\circ}$ of Theorem 1 below) holds true. So as to make this paper useful for further purposes we collect here all possible relations between different parts of the

\footnotetext{
${ }^{2}$ By the dimension of a Hilbert space we understand, as usual, the cardinality of an orthonormal basis. Thus saying "the dimension is equal to $\infty$ " means only that the space is not finite dimensional; we say this when we are not interested in what the cardinality in question is equal to.
} 
spectra of a subnormal operator and its minimal normal extension.

In the sequel we adopt the conventional notations $\sigma_{p}(S), \sigma_{a p}(S), \sigma_{c}(S)$ and $\sigma_{r}(S)$ for the point, approximate point, continuous and residual part of the spectrum $\sigma(S)$ of $S$, respectively.

Theorem 1. Let $S$ be a subnormal operator in $\mathscr{H}$. If $N$ is a minimal normal extension of spectral type of $S$, then

$$
\begin{aligned}
1^{\circ} & \sigma_{p}(S) \subset \sigma_{p}(N), \\
2^{\circ} & \sigma_{a p}(S) \subset \sigma_{a p}(N)=\sigma(N), \\
3^{\circ} & \sigma_{c}(S) \subset \sigma_{c}(N) \text { and } \sigma_{c}\left(S^{*}\right) \subset \sigma_{c}\left(N^{*}\right), \\
4^{\circ} & \sigma_{p}(N) \subset \sigma_{p}(\bar{S}) \cup \sigma_{r}(\bar{S}), \\
5^{\circ} & \sigma_{p}(N) \subset \sigma_{p}\left(S^{*}\right)^{*} \text { and } \sigma_{p}\left(N^{*}\right)=\sigma_{p}(N)^{*}, \\
6^{\circ} & \sigma_{c}(N) \subset \sigma_{r}(S) \cup \sigma_{c}(S) \text { and } \sigma_{c}\left(N^{*}\right)=\sigma_{c}(N)^{*}, \\
7^{\circ} & \sigma_{c}(N)^{*} \subset \sigma_{p}\left(S^{*}\right) \cup \sigma_{c}\left(S^{*}\right), \\
8^{\circ} & \sigma_{r}(N)=\sigma_{r}\left(N^{*}\right)=\sigma_{r}\left(S^{*}\right)=\varnothing, \\
9^{\circ} & \sigma(N) \subset \sigma(S), \\
10^{\circ} & \sigma_{a p}\left(N^{*}\right) \subset \sigma_{a p}\left(S^{*}\right)=\sigma\left(S^{*}\right) .
\end{aligned}
$$

Proof. Conclusions $1^{\circ}$ and $2^{\circ}$ hold trivially.

Now we proceed to the proof of $5^{\circ}$. First we show that

$$
\operatorname{ker} S^{*}=\{0\} \quad \text { implies } \operatorname{ker} N=\{0\} .
$$

Indeed, for an arbitrary $f \in \mathcal{K}, P E(\{0\}) f \in \operatorname{ker} S^{*}$, where $P$ is the orthogonal projection of $\mathcal{K}$ onto $\mathscr{H}$ and $E$ is the spectral measure of $N$ (to see this take $g \in \mathscr{D}(S)$ and notice that $\left.\langle\operatorname{Sg}, P E(\{0\}) f\rangle=\left\langle g, N^{*} E(\{0\}) f\right\rangle=0\right)$. Since ker $S^{*}=\{0\}$, $P E(\{0\}) f=0$ for each $f \in \mathcal{K}$. Thus $P E(\{0\})=0$. This implies that $P \perp E(\{0\})$ and consequently $\mathscr{H} \subset E(C \backslash\{0\}) \mathcal{K}$. Since $E(C \backslash\{0\}) \mathcal{K}$ reduces $N$, minimality of $N$ forces $E(C \backslash\{0\}) \mathcal{K}=\mathcal{K}$. So $E(\{0\})=0$. This means that $0 \in \mathbb{C} \backslash \sigma_{p}(N)$.

Take $\lambda \in C \backslash \sigma_{p}\left(S^{*}\right)$. This means that $\operatorname{ker}\left(\lambda-S^{*}\right)=\{0\}$. Since $\lambda^{*}-N$ is the minimal normal extension of $\lambda^{*}-S$, (2) implies that $\lambda^{*} \in \mathbb{C} \backslash \sigma_{p}(N)$. This completes the proof of the essential part of $5^{\circ}$.

Pass to the proof of $8^{\circ}$. To prove it notice that

$$
\sigma_{r}\left(A^{*}\right)=\varnothing \text { for a hyponormal } A
$$

(recall that $A$ is said to be hyponormal if $\mathscr{D}(A) \subset \mathscr{D}\left(A^{*}\right)$ and $\left\|A^{*} f\right\| \leqq\|A f\|$, $f \in \mathscr{D}(A))$. Indeed, if $\lambda \in \sigma_{r}\left(A^{*}\right)$, then $\operatorname{ker}\left(\lambda^{*}-\bar{A}\right)=\left(\left(\lambda-A^{*}\right) \mathscr{D}\left(A^{*}\right)\right)^{\perp} \neq\{0\}$. Since $\lambda^{*}-\bar{A}$ is hyponormal, $\operatorname{ker}\left(\lambda-A^{*}\right) \neq\{0\}$. This contradicts $\lambda \in \sigma_{r}\left(A^{*}\right)$. The conclusion $8^{\circ}$ follows from (3), because $N, N^{*}$ and $S$ are hyponormal. 
Now it is convenient to prove $9^{\circ}$. Without loss of generality we can assume $S$ is closed. Then we show, passing to resolvent sets, that $\rho(S) \subset \rho(N)$.

Suppose $0 \in \rho(S)$. Take $\varepsilon>0$ such that $\varepsilon\left\|S^{-1}\right\|<1$. Let $E$ denote the spectral measure of $N$. Take $h \in E\left(\Delta_{\varepsilon}\right) \mathcal{K}$ and $f \in \mathscr{H}$ (here $\Delta_{\varepsilon}$ is the open disc centered at 0 with radius $\varepsilon$ ). Since $S^{n} S^{-n} f=f$ for $n \geqq 0$, we get

$$
\begin{aligned}
|\langle h, f\rangle| & =\left|\left\langle h, S^{n} S^{-n} f\right\rangle\right|=\left|\left\langle h, N^{* n} S^{-n} f\right\rangle\right|=\left|\left\langle N^{n} h, S^{-n} f\right\rangle\right| \leqq\left\|N^{* n} h\right\|\left\|S^{-1}\right\|^{n}\|f\| \\
& =\left\{\int_{\Delta_{\varepsilon}}|\lambda|^{2 n}\langle E(d \lambda) h, h\rangle\right\}^{1 / 2}\left\|S^{-1}\right\|^{n}\|f\| \leqq\left(\varepsilon\left\|S^{-1}\right\|\right)^{n}\|h\|\|f\| \quad \text { for } \quad n \geqq 0 .
\end{aligned}
$$

Hence $\langle h, f\rangle=0$ and, because $h \in E\left(\Delta_{\varepsilon}\right) \mathcal{K}$ is arbitrary, $\mathscr{H} \subset E\left(C \backslash \Delta_{\varepsilon}\right) \mathcal{K}$. Since $E\left(C \backslash \Delta_{\varepsilon}\right) \mathcal{K}$ reduces $E$, minimality of $N$ implies $E\left(C \backslash \Delta_{\varepsilon}\right) \mathcal{K}=\mathcal{K}$, which means that $0 \in \rho(N)$.

Take an arbitrary $\lambda \in \rho(S)$. Then, since $\lambda-N$ is a minimal normal extension of $\lambda-S$ and $0 \in \rho(\lambda-S)$, we can apply the above procedure to get the conclusion $9^{\circ}$.

Now we pass to the proof of $3^{\circ}$. Take $\lambda \in \sigma_{c}(S)$. Since $(\lambda-S) \mathscr{D}(S)$ is dense in $\mathcal{H}, \operatorname{ker}\left(\lambda^{*}-S^{*}\right)=\{0\}$. Due to $5^{\circ}, \operatorname{ker}(\lambda-N)=\{0\}$. Notice that $\lambda \in \sigma(N)$. If not, there would be $\lambda \in\left(\boldsymbol{C} \backslash \sigma_{a p}(N)\right) \subset\left(C \backslash \sigma_{a p}(S)\right)$ (use $2^{\circ}$ ). This would mean that $\lambda \in \sigma_{c}(S) \cap\left(C \backslash \sigma_{a p}(S)\right)=\varnothing$ (contradiction). Consequently, due to $8^{\circ}, \lambda \in \sigma(N) \backslash$ $\sigma_{p}(N)=\sigma_{c}(N)$. Since

$$
\sigma_{c}\left(A^{*}\right)=\sigma_{c}(A)^{*}, \quad \text { for closed } A,
$$

we have $\sigma_{c}\left(S^{*}\right) \subset \sigma_{c}\left(N^{*}\right)$. This proves $3^{\circ}$.

The conclusion $4^{\circ}$ can be derived from $5^{\circ}, 8^{\circ}$ and (4) as follows $\sigma_{p}(N) \subset$ $\sigma_{p}\left(S^{*}\right)^{*}=\left(\sigma\left(S^{*}\right) \backslash \sigma_{c}\left(S^{*}\right)^{*}=\sigma(\bar{S}) \backslash \sigma_{c}(\bar{S})=\sigma_{p}(\bar{S}) \cup \sigma_{r}(\bar{S})\right.$.

To prove $6^{\circ}$, notice that, because of $9^{\circ}, \sigma_{c}(N) \subset \sigma(S)$. Using $1^{\circ}$ we can exclude $\sigma_{p}(S)$ from the right hand side of this inclusion so as to get the first part of conclusion $6^{\circ}$. The other is a special case of (4).

Employing $6^{\circ}$, the inclusion $\sigma_{r}(A)^{*} \subset \sigma_{p}\left(A^{*}\right)$ for a closed $A$, and (4) we get $\sigma_{c}(N)^{*} \subset \sigma_{r}(\bar{S})^{*} \cup \sigma_{c}(\bar{S})^{*} \subset \sigma_{p}\left(S^{*}\right) \cup \sigma_{c}(\bar{S})^{*}=\sigma_{p}\left(S^{*}\right) \cup \sigma_{c}\left(S^{*}\right)$. This is precisely $7^{\circ}$.

To prove $10^{\circ}$ observe that $\sigma_{a p}\left(A^{*}\right)=\sigma\left(A^{*}\right)$ for a hyponormal $A$ (use (3)). This and $9^{\circ}$ give us

$$
\sigma_{a p}\left(N^{*}\right)=\sigma(N)^{*} \subset \sigma(S)^{*}=\sigma\left(S^{*}\right)=\sigma_{a p}\left(S^{*}\right) .
$$

This completes the proof of Theorem 1 .

Notice that none of the inclusions appearing in the conclusion of Theorem 1 can be replaced by equality. The situation considered in Example 1 provides us with arguments that inclusions $1^{\circ}, 5^{\circ}, 6^{\circ}, 7^{\circ}, 9^{\circ}$ and $10^{\circ}$ may be strict. The

${ }^{3}$ Our proof of this inclusion is patterned on that of Halmos for bounded operators [12]. The same inclusion for unbounded operators, settled in a somewhat different way, has been proved by McDonald and Sundberg in [13]. However, their definition of subnormality is more restrictive than ours and also their proof is much longer. 
other cases may be strict too; this is the instance of the creation operator, cf. Section 16.

Theorem 1 exhausts all essential relations between different parts of the spectra, which may happen in our circumstances.

Corollary 1. For a subnormal operator $S, \sigma(S) \neq \varnothing$.

The proof uses Proposition 1 and the conclusion $9^{\circ}$ of Theorem 1 .

4. To continue our considerations of spectral properties of subnormal operators we prove here a fact which is well known in the bounded case [6].

Theorem 2. Let $S$ be a subnormal operator in $\mathscr{H}$ and $N$ be any normal extension of $S$ acting in $\mathcal{K}$. Suppose $\omega$ is a connected component of $C \backslash \sigma(N)$. Then either $\omega \cap \sigma(S)=\varnothing$ or $\omega \subset \sigma(S)$.

Proof. First we prove the following general fact

$$
\partial \sigma(A) \subset \sigma_{a p}(A) \text { for a closable } A \text {. }
$$

Suppose $A$ is closed. Take $\lambda \in \partial \sigma(A)$. Since $\sigma(A)$ is closed, there is a sequence $\lambda_{n} \in C \backslash \sigma(A)$ such that $\lambda_{n} \rightarrow \lambda$. First thing we want to show is that $\left\|\left(A-\lambda_{n}\right)^{-1}\right\|$ $\rightarrow \infty$ as $n \rightarrow \infty$. If not, we can assume, choosing a subsequence if necessary, that there is a positive number $c$ such that

$$
\left\|\left(A-\lambda_{n}\right)^{-1}\right\| \leqq c, \quad n \geqq 1 .
$$

This implies that

$$
\left\|\left(A-\lambda_{n}\right) f\right\| \geqq\left\|\left(A-\lambda_{n}\right)^{-1}\right\|^{-1}\|f\| \geqq c^{-1}\|f\|, \quad f \in \mathscr{D}(A),
$$

and consequently

$$
\|(A-\lambda) f\| \geqq c^{-1}\|f\|, \quad f \in \mathscr{D}(A) .
$$

On the other hand, inequality (6) and the von Neumann formula give us

$$
\left\|\left(A-\lambda_{n}\right)^{-1}-\left(A-\lambda_{m}\right)^{-1}\right\| \leqq c^{2}\left|\lambda_{n}-\lambda_{m}\right|, \quad m, n \geqq 1 .
$$

Since $\lambda_{n} \in \mathbb{C} \backslash \sigma(A)$, for an arbitrary $g \in \mathscr{H}$ there exists an $f_{n} \in \mathscr{D}(A)$ such that $g=\left(A-\lambda_{n}\right) f_{n}$. This means that

$$
\left\|f_{n}-f_{m}\right\|=\left\|\left[\left(A-\lambda_{n}\right)^{-1}-\left(A-\lambda_{m}\right)^{-1}\right] g\right\|
$$

and, due to (8), $\left\{f_{n}\right\}$ converges to some $f \in \mathscr{H}$. Since $A f_{n}=g+\lambda_{n} f_{n} \rightarrow g+\lambda f$ and since $A$ is closed $f \in \mathscr{D}(A)$ and $A f=g+\lambda f$. Thus $g=(A-\lambda) f$, which proves surjectivity of $A-\lambda$. This and (7) imply that $\lambda \in C \backslash \sigma(A)$, which, because $\sigma(A)$ is closed, contradicts our assumption. Consequently, this shows that $\left\|\left(A-\lambda_{n}\right)^{-1}\right\| \rightarrow \infty$ as $\mathrm{n} \rightarrow \infty$. The aforesaid enables us to choose $g_{n} \in \mathcal{H}$ such that 
$\left\|g_{n}\right\|=1$ and $\left\|\left(A-\lambda_{n}\right)^{-1} g_{n}\right\| \rightarrow \infty$ as $n \rightarrow \infty$. Setting $f_{n}=\left(A-\lambda_{n}\right)^{-1} g_{n}$ we obtain

$$
\left\|(A-\lambda)\left(\left\|f_{n}\right\|^{-1} f_{n}\right)\right\| \leqq\left\|f_{n}\right\|^{-1}\left\|\left(A-\lambda_{n}\right) f_{n}\right\|+\left|\lambda_{n}-\lambda\right| \leqq\left\|f_{n}\right\|^{-1}+\left|\lambda_{n}-\lambda\right| .
$$

Since $\left\|f_{n}\right\| \rightarrow \infty,(A-\lambda)\left(\left\|f_{n}\right\|^{-1} f_{n}\right) \rightarrow 0$. This proves the conclusion for closed $S$. If $S$ is not closed, the conclusion follows from the fact that $\sigma(A)=\sigma\left(A^{-}\right)$and $\sigma_{a p}(A)=\sigma_{a p}\left(A^{-}\right)$.

Now turn back to the proof of the Theorem. Let $\omega$ be a connected component of $C \backslash \sigma(S)$. Due to $2^{\circ}$ of Theorem 1 and (5) we have

$$
\partial \sigma(S) \subset \sigma_{a p}(S) \subset \sigma(N) .
$$

Thus $\omega \cap \sigma(S)=\omega \cap($ int $\sigma(S))$, which means that $\omega \cap \sigma(S)$ is open. But $\sigma(S)$ is closed, $\boldsymbol{\omega} \cap(\boldsymbol{C} \backslash \sigma(S))$ is always open. Connectivity of $\omega$ implies that either $\omega \cap \sigma(S)=\varnothing$ or $\omega \cap(C \backslash \sigma(S))=\varnothing$.

Corollary 2. Let $N$ be a minimal normal extension of spectral type of $S$. Then

$1^{\circ} \quad \partial \sigma(S) \subset \sigma_{a p}(S) \subset \sigma_{a p}(N)=\sigma(N) \subset \sigma(S)$,

$2^{\circ}$ if $\sigma(N)=\boldsymbol{C}$, then $\boldsymbol{\sigma}(S)=\boldsymbol{C}$,

$3^{\circ}$ if $S$ is maximal symmetric but not selfadjoint and $N$ is selfadjoint, then $\sigma(N)=\boldsymbol{R}$.

The part $3^{\circ}$ requires some comment. Since the spectrum of $S$ is precisely either the upper or the lower closed halfplane, $1^{\circ}$ establishes the conclusion of $3^{\circ}$.

5. Our next result bears a resemblance to Theorem 2.3 of [14].

Proposition 2. Let $N$ be a normal operator in $\mathcal{K}$ and $\mathcal{H}$ be closed linear subspace of $\mathcal{K}$. Then for any complex Borel function $\Phi$ defined on $\sigma(N)$ we have (according to notation of (1))

$$
\sigma\left(\Phi(N)_{\mathscr{H}}\right)=\sigma\left(\Phi\left(N_{\mathscr{H}}\right)\right) .
$$

Proof. First of all notice that since the space $\mathscr{A}_{s}[N]$ reduces $N$ to $N_{\mathscr{H}}$, $\sigma\left(N_{\mathscr{H}}\right) \subset \sigma(N)$ and the operator $\Phi\left(N_{\mathscr{H}}\right)$ is well defined.

Consider now the case $N=N_{\mathscr{H}}$. The inclusion $\sigma\left(\Phi(N)_{\mathscr{H}}\right) \subset \sigma(\Phi(N))$ is obvious. Now we want to prove the reverse inclusion. If $E$ is the spectral measure of $N$, then $E_{1}=E \circ \Phi^{-1}$ is a spectral measure of $\Phi(N)$ and $E_{2}=\left.E_{1}\right|_{\mathscr{H}_{s}[\Phi(N)]}$ is a spectral measure of $\Phi(N)_{\mathscr{r}}$. Suppose that there is $\lambda \in \sigma(\Phi(N)) \backslash \sigma\left(\Phi(N)_{\mathscr{H}}\right)$. Choose $\varepsilon>0$ such that $\operatorname{dist}\left(\lambda, \sigma\left(\Phi(N)_{\mathscr{r}}\right)\right)>2 \varepsilon$. Let $\omega=\sigma(\Phi(N)) \cap \Delta(\lambda ; \varepsilon)$, where $\Delta(\lambda ; \varepsilon)$ is an open disc of radius $\varepsilon$ and center $\lambda$. Then $\bar{\omega} \cap \sigma\left(\Phi(N)_{\mathscr{H}}\right)=\varnothing$. Thus $E\left(\Phi^{-1}(\bar{\omega})\right) f=0$ for each $f \in \mathscr{H}_{s}[\Phi(N)]$. This implies that $\mathscr{H}_{\subset} \mathscr{H}_{s}[\Phi(N)] \subset$ $\left(E\left(\Phi^{-1}(\bar{\omega})\right) \mathcal{K}\right)^{\perp}$. Since $\omega$ is relatively open in $\sigma(\Phi(N)), E\left(\Phi^{-1}(\bar{\omega})\right) \neq 0$. There- 
fore $\mathscr{H}_{C} \subset \mathcal{H}_{s}[\Phi(N)] \subset\left(E\left(\Phi^{-1}(\bar{\omega})\right) \mathcal{K}\right)^{\perp} \neq \mathcal{K}$. Since $\left(E\left(\Phi^{-1}(\bar{\omega})\right) \mathcal{K}\right)^{\perp}$ reduces $E$, it reduces $N$ too. All this contradicts $N=N_{\mathscr{H}}$.

The case of an arbitrary $N$ can be reduced to that just considered by the equality $\Phi\left(N_{\mathscr{H}}\right)=\Phi\left(N_{\mathscr{H}}\right)_{\mathfrak{H}}$. This completes the proof of Proposition 3.

6. Now we present the promised example.

Example 1. Take $\mathscr{K}=\mathcal{L}^{2}[a, b]$, where $a$ and $b$ are (finite) real numbers. Consider an operator $S$ with domain $\mathscr{D}(S)=\left\{f \in \mathcal{L}^{2}[a, b]: f\right.$ absolutely continuous on $[a, b], f^{\prime} \in \mathcal{L}^{2}[a, b]$ and $\left.f(a)=f(b)=0\right\}$, which is defined as

$$
S f=i f^{\prime}, \quad f \in \mathscr{D}(S) \text {. }
$$

Then $S$ is a closed symmetric operator with defect indices $(1,1)$. This operator has a selfadjoint extension in the space $\mathscr{H}$. However, we are interested ${ }^{4}$ in having an extension $N$ such that

$$
\mathscr{D}(S)=\mathscr{D}(N) \cap \mathscr{A} .
$$

In order to get such an extension take $\mathcal{K}=\mathcal{L}^{2}[c, d]$ where $c$ and $d$ are (finite) real numbers. For $t \in[0,2 \pi)$ consider an operator $N_{t}$ with domain $\mathscr{D}\left(N_{t}\right)=$ $\left\{f \in \mathcal{L}^{2}[c, d]: f\right.$ absolutely continuous on $[c, d], f^{\prime} \in \mathcal{L}^{2}[c, d]$ and $\left.f(c)=e^{\mathrm{i} t} f(d)\right\}$ defined as

$$
N_{t} f=\mathrm{i} f^{\prime}, \quad f \in \mathscr{D}\left(N_{t}\right) .
$$

The operator $N_{t}$ is selfadjoint. It is known (cf. [1, p. 160]) that

where

$$
\sigma\left(N_{t}\right)=\sigma_{p}\left(N_{t}\right)=\left\{\lambda_{t k}: k \in \mathbb{Z}\right\}
$$

$$
\lambda_{t k}=(t-2 k \pi)(d-c)^{-1}
$$

The eigenvalues $\lambda_{t k}$ of $N_{t}$ are of multiplicity one and the coresponding eigen vectors $f_{t k}$ are

$$
f_{t k}(x)=(d-c)^{-1 / 2} \exp \left(-\mathrm{i} \lambda_{t k} x\right), \quad x \in[c, d] .
$$

So the spectral measure $E_{t}$ of $N_{t}$ is

$$
E_{t}(\sigma) f=\sum_{\lambda_{k} \in \sigma}\left\langle f, f_{t k}\right\rangle f_{t k}
$$

where $\sigma$ is a Borel subset of $\boldsymbol{R}$ and $f$ is in $\mathcal{L}^{2}[c, d]$. Fix now the intervals in such a way that $[a, b] \subset[c, d]$ and $d-c=2(b-a)$. Then $S \subset N_{t}$ and

$$
\mathscr{D}(S)=\mathscr{D}\left(N_{t}\right) \cap \mathscr{K} .
$$

We show that, for $t \in(0, \pi) \cup(\pi, 2 \pi), N_{t}$ is a minimal normal extension of $S$. To see this it is enough to prove, due to (9), that

${ }^{4}$ For some discussion concerning this equality see [19]. 


$$
\left\langle x_{[a, b]}, f_{t k}\right\rangle \neq 0, \quad \text { for every } k \in \boldsymbol{Z}
$$

where $x_{[a, b]}$ stands for the characteristic function of $[a, b]$. If not, then $\left\langle x_{[a, b]}, f_{t k}\right\rangle=0$ for some $k$ would imply that there existed $\iota \in \boldsymbol{Z}$ such that

$$
t=2 k \pi+2: \pi(b-a)(d-c)^{-1}=(2 k+\iota) \pi .
$$

This contradicts $t \in(0, \pi) \cup(\pi, 2 \pi)$.

Summarizing, we have an example of a symmetric (read: subnormal) operator having a plenty of (after fixing $[c, d]$ ) selfadjoint (read: normal) extensions $N_{t}$ of $S, t \in(0, \pi) \cup(\pi, 2 \pi)$, which

$1^{\circ}$ are minimal of spectral type,

$2^{\circ}$ have disjoint spectra,

$3^{\circ}$ are not equivalent,

$4^{\circ}$ satisfy equality (10).

All the informations about the spectra of $S$ and its extensions $N_{t}$, which can be derived from the above, are collected in the following table. This permits to make the spectral relations appearing here transparent.

\begin{tabular}{|c|c|c|c|c|c|}
\hline & $\sigma_{p}$ & $\sigma_{a p}$ & $\sigma_{r}$ & $\sigma_{c}$ & $\sigma$ \\
\hline$S$ & $\varnothing$ & $\varnothing$ & $\boldsymbol{C}$ & $\varnothing$ & $\boldsymbol{C}$ \\
\hline$N$ & $\Lambda$ & $\Lambda$ & $\varnothing$ & $\varnothing$ & $\Lambda$ \\
\hline$S^{*}$ & $\boldsymbol{C}$ & $\boldsymbol{C}$ & $\varnothing$ & $\varnothing$ & $\boldsymbol{C}$ \\
\hline$N^{*}$ & $\Lambda$ & $\Lambda$ & $\varnothing$ & $\varnothing$ & $\Lambda$ \\
\hline
\end{tabular}

Here $N=N_{t}$ and $\Lambda=\Lambda_{t}=\left\{\lambda_{t k}: k \in \boldsymbol{Z}\right\}$.

The operator $S$ just considered has a disadvantage; namely it has a selfadjoint extension in the initial space $\mathscr{A}$. If one would like to have a subnormal operator which has no normal extension in the initial space $\mathscr{H}$ (which is the case for bounded subnormals) and, on the other hand, which still has properties $1^{\circ}, 3^{\circ}$ and $4^{\circ}$, the simplest example would be a subnormal operator of the form $S \oplus V$, where $S$ is the operator just defined and $V$ is, say, the unilateral shift.

\section{Minimality of Cyclic Type}

7. Recall that a densely defined operator $N$ is said to be formally normal if

$$
\mathscr{D}(N) \subset \mathscr{D}\left(N^{*}\right) \text { and }\|N f\|=\left\|N^{*} f\right\| \text { for } f \in \mathscr{D}(N) .
$$

A densely defined operator $S$ in $\mathscr{H}$ is said to be formally subnormal if there exists another Hilbert space $\mathcal{K}$ containing $\mathscr{H}$ and a densely defined formally normal operator $N$ in $\mathcal{K}$ such that 


$$
\mathscr{D}(S) \subset \mathscr{D}(N) \cap \mathscr{K} \quad \text { and } \quad S f=N f, f \in \mathscr{D}(S) \text {. }
$$

It is known [7] that a formally normal operator need not be subnormal.

From now we assume that the operator $S$ leaves its domain invariant, i.e.

$$
S \mathscr{D}(S) \subset \mathscr{D}(S) \text {. }
$$

Within this class of operators, $S$ is formally subnormal if and only if it satisfies the so called Halmos-Bram condition [18, Prop. 2]. Moreover we can choose a formally normal extension $N$ of $S$ to satisfy

$$
\mathscr{D}(N)=\operatorname{lin}\left\{N^{* n} f: f \in \mathscr{D}(S), n \geqq 0\right\} .
$$

This justifies the following definition: a formally normal extension $N$ of $S$ is said to be minimal of cyclic type if

where

$$
N=\left(N i_{\mathscr{H}_{c}(N)}\right)^{-},
$$

$$
\mathscr{A}_{c}(N)=\operatorname{lin}\left\{N^{* n} f: f \in \mathscr{D}(S), n \geqq 0\right\} .
$$

The following notation will be useful in the sequel

$$
\mathscr{H}_{c}[N]=\mathscr{H}_{c}(N)^{-} .
$$

Proposition 3. Every formally subnormal operator satisfying (11) always has a minimal formally normal extension of cyclic type. Moreover, two formally normal extensions $N_{1}$ and $N_{2}$ of $S$, which are minimal of cyclic type, are $\mathscr{A}$-equivalent, i.e. there is a unitary operator $U: \mathcal{K}_{1} \rightarrow \mathcal{K}_{2}$ such that

$$
U f=f, f \in \mathscr{H} \text { and } U N_{1}=N_{2} U \text {. }
$$

Indeed, the formula $U N_{1}^{* n} f=N_{2}^{* n} f$ uniquely defines the unitary operator which meets the requirement.

Proposition 4. An operator $S$ (satisfying (11)) is subnormal if and only if it is formally subnormal and has a minimal formally normal extension of cyclic type, which is subnormal.

Proof. Let $M$ be a normal extension of $S$. Then $N=\left(\left.M\right|_{\mathscr{H}_{c}(M)}\right)^{-}$is a formally normal extension of $S$, which is minimal of cyclic type, acting in $\mathscr{H}_{c}[M]$ (cf. (12)). The converse is trivial.

Notice that, in general, $\left(\left.M\right|_{\mathscr{T}_{C}(M)}\right)^{-}$need not be minimal of spectral type even if $M$ is minimal of spectral type (Example 1 provides us with the argument; $\mathscr{H}_{c}(S)=\mathscr{D}(S)$ for a symmetric $S$ ). In other words, there are subnormal operators having no minimal normal extension of cyclic type. 
Theorem 3. Let $S$ be a subnormal operator satisfying (11). Suppose that it has at least one minimal normal extension of cyclic type. Then an arbitrary normal extension of $S$ is minimal of spectral type if and only if it is minimal of cyclic type.

Proof. Let $N$ be a minimal normal extension of $S$ of cyclic type in $\mathcal{K}$. What we want to show is $\mathcal{K}=\mathscr{H}_{s}[N]$. Take $g \in \mathcal{K} \ominus \mathcal{H}_{s}[N]$ and $f \in \mathscr{D}(S)$. Then, since $E(\sigma) f$ is in $\mathscr{H}_{s}[N],\langle E(\sigma) f, g\rangle=0$ for an arbitrary Borel set $\sigma$. This implies

$$
\left\langle N^{* n} f, g\right\rangle=\lim _{m \rightarrow \infty} \int_{|z|<m} z^{* n}\langle E(d z) f, g\rangle=0 .
$$

Consequently, $g \perp \mathscr{A}_{c}[N]=\mathcal{K}$. So $g=0$.

On the other hand, suppose $M$ is a minimal normal extension of spectral type. Then $M_{c}=\left(\left.M\right|_{\mathscr{H}_{c}(M)}\right)^{-}$is a formally normal extension of $S$, which is minimal of cyclic type. Since so is $N=\left(\left.N\right|_{\mathscr{H}_{c}(N)}\right)^{-}$, Proposition 3 gives us a unitary operator $U: \mathscr{H}_{c}[M] \rightarrow \mathscr{H}_{c}[N]$ such that $M_{c}=U^{-1} N U$. Since $N$ is normal, $M_{c}$ is normal too. This means that $\mathscr{H}_{\subset} \mathscr{H}_{c}[M]$ and $\mathscr{H}_{c}[M]$ reduces $M$. Spectral minimality of $M$ forces $\mathscr{H}_{c}[M]=\mathscr{H}_{s}[M]$. This completes the proof.

Corollary 3. If $S$ has at least one minimal normal extension of cyclic type, then all its minimal normal extensions of spectral type (they always have to exist, $c f$. Proposition $1(\alpha)$ ) are $\mathscr{H}$-equivalent.

8. Now we wish to focus our interst on a class of subnormal operators which always have minimal normal extensions of cyclic type. Recall that a vector $f \in \mathscr{D}(S)$ is said to be quasianalytic if

$$
\sum_{n=1}^{\infty}\left\|S^{n} f\right\|^{-1 / n}=\infty \text {. }
$$

Denote by $Q(S)$ the collection of all quasianalytic vectors of $S$.

Theorem 4. Let $S$ be a formally subnormal operator satisfying (11). If $\operatorname{lin} Q(S)=\mathscr{D}(S)$, then $S$ has a minimal normal extension of cyclic type.

Sketch of proof. ${ }^{5}$ Consider a formally normal extension $N$ of $S$, which is minimal of cyclic type (by Proposition 3 such an extension always exists). Denote by $N_{0}=\left.N\right|_{\mathscr{H}_{c}(N)}$. Notice that $Q(S) \subset Q\left(N_{0}\right)$ and, due to [17, Prop. 2], $N^{* n} f \in Q\left(N_{0}\right)$ for every $f \in Q(S)$. This implies that $\mathscr{D}\left(N_{0}\right)=\operatorname{lin} Q\left(N_{0}\right)$. Then, by [17, Theorem 1], $N^{\prime}=N_{0}^{-}$is the wanted normal extension of $S$.

9. A densely defined operator $S$ in $\mathscr{H}$ is said to be cyclic if there is a

\footnotetext{
${ }^{5}$ All the details can be found in [17, Theorem 8 and Remark 8$]$.
} 
vector (called a cyclic vector of $S$ ) $f_{0} \in \mathscr{D}^{\infty}(S)=\bigcap_{n=0}^{\infty} \mathscr{D}\left(S^{n}\right)$ such that $\mathscr{D}(S)=$ $\operatorname{lin}\left\{S^{n} f_{0}: n \geqq 0\right\}$. We have already shown [18, Prop. 3] that such an $S$ is subnormal if and only if there exists a non-negative measure $m$ on $C$ (call it a representing measure of $S$ ) such that

$$
\begin{gathered}
\int_{C}|z|^{2 n} \mathfrak{m}(d z)<\infty \quad \text { for every } \quad n \geqq 0, \\
\left\langle S^{m} f_{0}, S^{n} f_{0}\right\rangle=\int_{C} z^{m} z^{* n} \mathfrak{m}(d z) \quad \text { for all } m, n \geqq 0 .
\end{gathered}
$$

For any minimal normal extension $N$ of spectral type of $S$, we have a positive Borel measure $\mathfrak{m}_{N}$ on $C$ defined by

$$
\mathfrak{m}_{N}(\sigma)=\left\langle E(\sigma) f_{0}, f_{0}\right\rangle, \quad \sigma \text { a Borel set, }
$$

where $E$ is the spectral measure of $N$.

On the other hand, given a positive Borel measure $\mathfrak{m}$ on $\mathbb{C}$ satisfying (13), we can define the operator $N_{\mathfrak{m}}$ of multiplication by the independent variable in $\mathcal{L}^{2}(\mathfrak{m})$, which is a minimal normal extension of $S_{\mathfrak{m}}=\left.N_{\mathfrak{m}}\right|_{C[z]}$ of spectral type. This means that the operator $S_{\mathfrak{m}}$ acting in $\mathscr{H}_{\mathfrak{m}}$, the $\mathcal{L}^{2}(\mathfrak{m})$-closure of $C[z]$, is subnormal.

The following result shows, among other things, that if $\mathfrak{m}$ is a representing measure of $S$, then $S$ is equivalent to $S_{\mathfrak{m}}$. So the operator $S_{\mathfrak{m}}$ can be viewed as a functional model of $S$.

Theorem 5. Let $S$ be a cyclic subnormal operator in $\mathscr{A}$ with the cyclic vector $f_{0}$. Then

$1^{\circ}$ for each minimal normal extension $N$ of spectral type of $S, \mathfrak{m}=\mathfrak{m}_{N}$ is a representing measure of $S$,

$2^{\circ} \quad \mathfrak{m}_{N_{1}}=\mathfrak{m}_{N_{2}}$ if and only if $N_{1}$ and $N_{2}$ are $\mathcal{H}$-equivalent,

$3^{\circ}$ for any representing measure $\mathfrak{m}$ of $S, S$ is equivalent to $S_{\mathfrak{m}}$,

$4^{\circ}$ for any representing measure $\mathfrak{m}$ of $S$ there is a minimal normal extension $N$ of spectral type of $S$ such that $\mathfrak{m}=\mathfrak{m}_{N}$,

$5^{\circ}$ for each minimal normal extension $N$ of spectral type of $S, \sigma(N)=\operatorname{supp}_{N}$.

Proof. $1^{\circ}$ is obvious. To prove $2^{\circ}$ suppose $N_{1}$ and $N_{2}$ are minimal normal extensions of spectral type of $S$ acting in $\mathcal{K}_{1}$ and $\mathcal{K}_{2}$, respectively, such that $\mathfrak{m}_{N_{1}}=\mathfrak{m}_{N_{2}}$. Denote by $E_{1}$ and $E_{2}$ their spectral measures. Since $E_{1}$ and $E_{2}$ are spectral measures, standard argument applied to $\left\langle E_{1}(\sigma) f_{0}, f_{0}\right\rangle=\left\langle E_{2}(\sigma) f_{0}, f_{0}\right\rangle$ gives us

$$
\left\langle E_{1}(\sigma) f_{0}, E_{1}(\rho) f_{0}\right\rangle=\left\langle E_{2}(\sigma) f_{0}, E_{2}(\rho) f_{0}\right\rangle \text {. }
$$

Notice that $E_{\imath}(\rho) S^{n} f_{0}=\int_{\rho} z^{n} E_{\imath}(d z) f_{0} \in \operatorname{clolin}\left\{E_{\imath}(\sigma) f_{0}: \sigma\right.$ a Borel set $\}$, for an arbitrary Borel subset $\rho$ of $C$. Since $\mathcal{K}_{\imath}=\mathscr{H}_{s}\left[N_{\imath}\right]$ and $S$ is cyclic we get 


$$
\mathcal{K}_{\imath}=\operatorname{clolin}\left\{E_{\imath}(\sigma) f_{0}: \sigma \text { a Borel set }\right\} .
$$

Due to (16) and (17), there is precisely one unitary operator $U$ from $\mathcal{K}_{1}$ to $\mathcal{K}_{2}$ such that $U E_{1}(\sigma) f_{0}=E_{2}(\sigma) f_{0}$. This and (17) implies that $U E_{1}(\sigma)=E_{2}(\sigma) U$. This, in turn, implies that $U N_{1}^{n}=N_{2}^{n} U, n \geqq 1$. Thus, since $U f_{0}=f_{0}$, we have $U S^{n} f_{0}=U N_{1}^{n} f_{0}=N_{2}^{n} U f_{0}=N_{2}^{n} f_{0}=S^{n} f_{0}$. Since $f_{0}$ is cyclic, we get $\left.U\right|_{\mathscr{H}}=I_{\mathscr{H}}$ which means that $N_{1}$ and $N_{2}$ are $\mathscr{K}$-equivalent.

The proof of the reverse implication of $2^{\circ}$ is straightforward.

To prove $3^{\circ}$ notice that, due to (14), there is precisely one unitary operator $V$ from $\mathscr{H}_{\text {to }} \mathscr{H}_{\mathfrak{m}}$ such that $V S^{n} f_{0}=z^{n}, n \geqq 0$ and $V S=S_{\mathfrak{m}} V$, which means that $S$ and $S_{\mathfrak{m}}$ are equivalent.

The operator $N=N_{\mathrm{m}}$ meets what is required in $4^{\circ}$.

Let $E$ be the spectral measure of a minimal normal extension $N$ (acting in $\mathcal{K})$ of spectral type of $S$. Then, for an open set $\sigma, \mathfrak{m}_{N}(\sigma)=0$ if and only if $E(\sigma)=0$. This follows from (15) and from the fact that $\mathcal{K}=\operatorname{clolin}\left\{E(\sigma) f_{0}: \sigma\right.$ a Borel set\} using the same kind of argument as in the proof of $2^{\circ}$. This gives us the conclusion $5^{\circ}$.

Corollary 4. Under assumptions of Theorem 5 the operator $S$ has as many non $\mathcal{H}$-equivalent minimal normal extensions of spectral type as the complex moment sequence $\left\{\left\langle S^{m} f_{0}, S^{n} f_{0}\right\rangle\right\}_{m, n}$ has representing measures (i.e. measures $\mathfrak{m}$ satisfying (14)).

It might be interesting to know when there exists a minimal normal extension of cyclic type of a given cyclic subnormal operator.

Proposition 5. Under assumptions of Theorem 5 the operator $S$ has at least one minimal normal extension of cyclic type if and only if there is a positive Borel measure $\mathfrak{m}$ satisfying (13), (14) and such that $\boldsymbol{C}\left[z, z^{*}\right]$ is dense in $\mathcal{L}^{2}\left(\left(1+|z|^{2}\right) \mathfrak{m}\right)^{6}$.

Due to Theorem 3, the proof of Proposition 5 is based on the observation that the $\mathcal{L}^{2}\left(\left(1+|z|^{2}\right) \mathfrak{m}\right)$-closure of $C\left[z, z^{*}\right]$ is equal to $\mathcal{L}^{2}\left(\left(1+|z|^{2}\right) \mathfrak{m}\right)$ if and only if $N_{\mathfrak{m}}=\left(\left.N_{\mathfrak{m}}\right|_{c\left[z, z^{*}\right]}\right)^{-}$, where $N_{\mathfrak{m}}$ is the operator of multiplication by $z$ in $\mathcal{L}^{2}(\mathfrak{m})$.

Corollary 5. Under the assumptions of Theorem 5 the operator $S$ has at least one minimal normal extension of cyclic type if there is a positive Borel measure $\mathfrak{m}$ satisfying (13), (14) and such that $C\left[z, z^{*}\right]$ is dense in $\mathcal{L}^{p}(\mathfrak{m})$ for some $p>2$.

This follows from Proposition 5 and [11, Th. 10].

${ }_{6}$ This corresponds to Theorem 8 of [11] where so-called ultradeterminancy of the multidimensional moment problem has been considered. 
The example discussed in [11, Remark 4, p. 59] can provide us with an indeterminate measure $\mathfrak{m}$ such that $C\left[z, z^{*}\right]$ is not dense in $\mathcal{L}^{2}\left(\left(1+|z|^{2}\right) \mathfrak{m}\right)$. Thus, in virtue of Proposition 5, the operator of multiplication by $z$ defined on $\mathbb{C}[z]$ has no normal extension of cyclic type.

\section{Analytic Models for Cyclic Operators}

10. Let $S$ be a cyclic operator in $\mathscr{H}$ with the cyclic vector $f_{0}$.

Lemma 1. If $S$ is a cyclic operator in $\mathscr{H}$ and $\operatorname{dim} \mathscr{H}=\infty$, then the set of its cyclic vectors forms a one dimensional space.

Proof. Let $f_{0}$ be a cyclic vector of $S$. Since $\mathcal{H}$ is infinite dimensional, then the linear map $\Phi: \mathscr{D}(S) \rightarrow C[z]$ given by $\Phi\left(p(S) f_{0}\right)=p$ is a linear isomorphism such that $M_{z} \Phi=\Phi S$ where $M_{z}$ is the operator of multiplication by $z$ in $C[z]$. Since the set of cyclic vectors for $M_{z}$ is composed of polynomials of degree zero, we get the conclusion.

Lemma 2. $\lambda \in \sigma_{p}\left(S^{*}\right)^{*}$ if and only if there is $c_{\lambda}>0$ such that

$$
|p(\lambda)| \leqq c_{\lambda}\left\|p(S) f_{0}\right\|
$$

for any polynomial $p \in C[z]$. If this happens, then $\operatorname{dim}\left(\operatorname{ker}\left(\lambda^{*}-S^{*}\right)\right)=1$.

Proof. Suppose that $\lambda \in \sigma_{p}\left(S^{*}\right)^{*}$. This means that there is $k_{\lambda} \in \mathscr{M}$ such that $S^{*} k_{\lambda}=\lambda^{*} k_{\lambda}$ and $\left\|k_{\lambda}\right\|=1$. Then we get $p\left(S^{*}\right) k_{\lambda}=p\left(\lambda^{*}\right) k_{\lambda}$ for $p \in \boldsymbol{C}[z]$. Since $p\left(S^{*}\right) \subset\left(p^{*}(S)\right)^{*}$, where $p^{*}(z)=\left(p\left(z^{*}\right)\right)^{*}$ for $z \in C$, we have $p(S)^{*} k_{\lambda}=$ $p^{*}\left(\lambda^{*}\right) k_{\lambda}=p(\lambda)^{*} k_{\lambda}$. This gives us

$$
\left\langle p(S) f_{0}, k_{\lambda}\right\rangle=p(\lambda)\left\langle f_{0}, k_{\lambda}\right\rangle, \quad p \in C[z] .
$$

Since $S$ is cyclic, equality (19) implies $\left\langle f_{0}, k_{\lambda}\right\rangle \neq 0$. Put $c_{\lambda}=\left|\left\langle f_{0}, k_{\lambda}\right\rangle\right|^{-1}$. Thus, using again (19), we get (18).

Asumming (18) and applying the Riesz representation theorem to the functional $p(S) f_{0} \rightarrow p(\lambda)$, one can find $h_{\lambda} \neq 0$ such that $p(\lambda)=\left\langle p(S) f_{0}, h_{\lambda}\right\rangle$. This gives us $\left\langle S p(S) f_{0}, h_{\lambda}\right\rangle=\lambda p(\lambda)=\left\langle p(S) f_{0}, \lambda^{*} h_{\lambda}\right\rangle, p \in C[z]$. Because $S$ is cyclic, $h_{\lambda} \in \mathscr{D}\left(S^{*}\right)$ and $S^{*} h_{\lambda}=\lambda^{*} h_{\lambda}$. This means that $\lambda^{*} \in \sigma_{p}\left(S^{*}\right)$.

Take non-zero vectors $h_{\imath} \in \operatorname{ker}\left(\lambda^{*}-S^{*}\right), i=1,2$. Then, using the same kind of argument as in the proof of the "only if" part, we have $\left\langle p(S) f_{0}, h_{2}\right\rangle=$ $p(\lambda)\left\langle f_{0}, h_{2}\right\rangle, \rho \in \boldsymbol{C}[z]$. This forces that $h_{1}=\alpha h_{2}$, where $\alpha=\left\langle h_{1}, f_{0}\right\rangle\left\langle h_{2}, f_{0}\right\rangle^{-1}$, completing the proof.

Assume for the moment that $\mathscr{H}$ is not finite dimensional and $\left\|f_{0}\right\|=1$. Since $S$ is cyclic this is equivalent to the fact that $\left\{S^{n} f_{0}\right\}_{n \geqq 0}$ is composed of linearly independent vectors. Now let $\left\{e_{n}\right\}_{n \geqq 0}$ be an orthonormal basis in $\mathscr{H}$ 
and let $\left\{r_{n}\right\}_{n \geqq 0}$ be a sequence of polynomials from $C[z]$ such that

$$
e_{n}=r_{n}(S) f_{0},
$$

and

$$
\operatorname{lin}\left\{e_{n}\right\}_{n \geqq 0}=\operatorname{lin}\left\{S^{n} f_{0}\right\}_{n \geqq 0} .
$$

One can get such a sequence $\left\{r_{n}\right\}_{n \geqq 0}$ applying the Gram-Schmidt orthonormalization procedure to $\left\{S^{n} f_{0}\right\}_{n \geqq 0}$. The polynomials $r_{n}$ can be given explicitely by $r_{0} \equiv 1$,

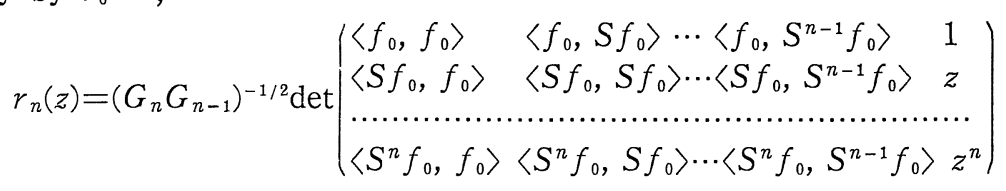

$G_{n}=\operatorname{det}\left(\left\langle S^{i} f_{0}, S^{\jmath} f_{0}\right\rangle\right)_{2, j=0}^{n}$ for $n \geqq 1$ and $G_{0}=1$.

Proposition 6. Suppose $\operatorname{dim} \mathscr{H}=\infty$ and $S$ is a cyclic operator in $\mathscr{H}$ with the cyclic vector $f_{0}$. If the orthonormal basis $\left\{e_{n}\right\}_{n \geqq 0}$ in $\mathscr{H}$ and the sequence $\left\{r_{n}\right\}_{n \geqq 0}$ of polynomials in $C[z]$ satisfy (20) and (21), then the following conditions are equivalent:

$1^{\circ} \quad \lambda^{*} \in \sigma_{p}\left(S^{*}\right)$,

$2^{\circ}$ there is $c_{\lambda}>0$ such that $|p(\lambda)| \leqq c_{\lambda}\left\|p(S) f_{0}\right\|$ for any polynomial $p \in C[z]$,

$3^{\circ}$ there is (precisely one) vector $h_{\lambda} \in \mathscr{K}$ such that $p(\lambda)=\left\langle p(S) f_{0}, h_{\lambda}\right\rangle, p \in \boldsymbol{C}[z]$,

$4^{\circ} \quad \sum_{n=0}^{\infty}\left|r_{n}(\lambda)\right|^{2}<\infty$.

If this happens, then

$5^{\circ} \quad h_{\lambda}=\sum_{n=0}^{\infty} r_{n}(\lambda)^{*} e_{n},\left\|h_{\lambda}\right\|^{2}=\sum_{n=0}^{\infty}\left|r_{n}(\lambda)\right|^{2}$ and $S^{*} h_{\lambda}=\lambda^{*} h_{\lambda}$,

$6^{\circ}$ if $\tilde{f}_{0}$ is another cyclic vector of $S$, then the vector $\tilde{h}_{\lambda}$ corresponding to it (via $\left.3^{\circ}\right)$ is equal to $\left\langle\tilde{f}_{0}, f_{0}\right\rangle\left\|\tilde{f}_{0}\right\|^{-2} h_{\lambda}$.

Proof. $1^{\circ} \Leftrightarrow 2^{\circ}$ due to Lemma $2.2^{\circ} \Leftrightarrow 3^{\circ}$ by the Riesz representation theorem $\left(c_{\lambda}=\left\|h_{\lambda}\right\|\right)$.

$3^{\circ} \Rightarrow 4^{\circ}$. Using (20) and the Parseval equality we get

$$
\sum_{n=0}^{\infty}\left|r_{n}(\lambda)\right|^{2}=\left\|h_{\lambda}\right\|^{2}<\infty .
$$

$4^{\circ} \Rightarrow 3^{\circ}$. Since $\operatorname{dim} \mathscr{H}$ is infinite, $\left\{S^{n} f_{0}\right\}_{n \geqq 0}$ is composed of linearly independent vectors. This implies that $p(S) f_{0}=0$ if and only if $p=0, p \in C[z]$. This, (20) and (21) guarantees that $\left\{r_{n}\right\}_{n \geqq 0}$ is the Hamel basis for $\boldsymbol{C}[z]$. Define $h_{\lambda}=\sum_{n=0}^{\infty} r_{n}(\lambda) * e_{n}$. Take an arbitrary polynomial $p \in C[z]$. Then there are complex numbers $\alpha_{0}, \cdots, \alpha_{n}$ such that $p=\sum_{k=0}^{n} \alpha_{k} r_{k}$. Thus 


$$
\left\langle p(S) f_{0}, h_{\lambda}\right\rangle=\sum_{k=0}^{n} \alpha_{k}\left\langle r_{k}(S) f_{0}, h_{\lambda}\right\rangle=\sum_{k=0}^{n} \alpha_{k} r_{k}(\lambda)=p(\lambda) .
$$

The proof of $5^{\circ}$ is included implicitly in $4^{\circ} \Rightarrow 3^{\circ}$.

The conclusion $6^{\circ}$ follows from Lemma 1 , by direct calculation. This completes the proof.

Notice that Proposition 6 fails on dropping (21).

Remark 1. It is easy to see that if $S$ is a cyclic subnormal operator with a representing measure $\mathfrak{m}$, then $\operatorname{dim} \mathscr{H}=\aleph_{0}$ is equivalent to the fact that supp $\mathfrak{m}$ is at least countable.

Denote by $\kappa_{s}$ the function defined on $C$ as

$$
\kappa_{s}(\lambda)=\left\{\begin{array}{cl}
\infty & \text { if } \lambda \text { is not in } \sigma_{p}\left(S^{*}\right)^{*} \\
\left\|h_{\lambda}\right\|^{2} & \text { if } \lambda \text { is in } \sigma_{p}\left(S^{*}\right)^{*}
\end{array}\right.
$$

where $h_{\lambda}$ is as in Proposition 6. Then Proposition 6 implies

$$
\begin{gathered}
\kappa_{s}(\lambda)=\sum_{n=0}^{\infty}\left|r_{n}(\lambda)\right|^{2}, \\
\sigma_{p}\left(S^{*}\right)^{*}=\left\{\lambda: \kappa_{s}(\lambda)<\infty\right\}
\end{gathered}
$$

and, for any $\lambda$,

$$
|p(\lambda)|^{2} \leqq c\left\|p(S) f_{0}\right\|^{2} \quad \text { for all } \quad p \in \mathbb{C}[z] \Leftrightarrow \kappa_{s}(\lambda) \leqq c .
$$

The function $\kappa_{s}$ depends on the particular choice of a cyclic vector $f_{0}$ and the way it does is described in conclusion $6^{\circ}$ of Proposition 6.

Corollary 6. For a cyclic operator $S, \kappa_{s}$ is lower semicontinuous. Moreover, if $\omega$ is a bounded open set such that $\kappa_{s}(\lambda) \leqq c$ for $\lambda \in \partial \omega$ with some $c>0$, then $\kappa_{s}(\lambda) \leqq c$ for $\lambda \in \omega^{-}$.

Proof. Since

$$
\left\{\lambda \in C: \kappa_{s}(\lambda) \leqq c\right\}=\bigcap_{k=0}^{\infty}\left\{\lambda \in C: \sum_{n=0}^{k}\left|\gamma_{n}(\lambda)\right|^{2} \leqq c\right\},
$$

the function $\kappa_{s}$ is lower semicontinuous.

Condition (24) implies $|p(\lambda)|^{2} \leqq c\left\|p(S) f_{0}\right\|^{2}$ for all $p \in C[z]$ and $\lambda \in \partial \omega$. The maximum modulus principle for holomorphic functions ensures us that $|\rho(\lambda)|^{2} \leqq$ $c\left\|p(S) f_{0}\right\|^{2}$ for all $p \in \mathbb{C}[z]$ and $\lambda \in \omega^{-}$. Using again (24) we get the final conclusion.

Corollary 7. For a cyclic operator $S, \sigma_{p}\left(S^{*}\right)$ is a union of an increasing sequence of compact sets having no bounded holes. 
Proof. The case $\operatorname{dim} \mathscr{K}<\infty$ is trivial. In the other case it follows from Proposition 6 that

$$
\sigma_{p}\left(S^{*}\right) *=\bigcup_{m=0}^{\infty} \sigma_{m}
$$

where $\sigma_{m}=\left\{\lambda \in C: \kappa_{s}(\lambda) \leqq m\right\} \cap \operatorname{disc}(0 ; m)^{-}$. Suppose some $\sigma_{m}$ has a bounded hole $\omega$. Then $\partial \omega \subset \sigma_{m}$ and, consequently, $\kappa_{s}(\lambda) \leqq m$ for $\lambda \in \partial \omega$. By Corollary 6 , $\kappa_{s}(\lambda) \leqq m$ for $\lambda \in \omega$. This means that $\omega \subset \sigma_{m}$ which gives us contradiction.

11. The next result shows that the converse direction in Proposition 6 is possible.

Proposition 7. Suppose we are given a sequence of polynomials $\left\{r_{n}\right\}_{n \geqq 0}$, being a Hamel basis for $C[z]$. Then there is a cyclic operator $S$ in some Hilbert space $\mathscr{A}$ with the cyclic vector $f_{0}$ such that $\sigma_{p}\left(S^{*}\right)^{*}=\left\{\lambda: \sum_{n=0}^{\infty}\left\{\left.r_{n}(\lambda)\right|^{2}<\infty\right\}\right.$ and the function $\kappa_{s}$ satisfies (22) and (23).

Proof. Since $\left\{r_{n}\right\}_{n \geq 0}$ is a Hamel basis of $C[z]$, there is precisely one scalar product such that $\left\langle r_{m}, r_{n}\right\rangle=\delta_{m, n}$. Denote by $\mathscr{f}$ the completion of this inner product space. Let $S$ be the operator of multiplication by $z$ in $C[z]$. Then $S$ is cyclic with the cyclic vector $f_{0}=r_{0}$ and $p(S) f_{0}=p$ for $p \in \boldsymbol{C}[z]$. In particular $r_{n}(S) f_{0}=r_{n}$. Thus we are in the starting point (with $e_{n}=r_{n}$ ) of Proposition 6.

One can choose a sequence $\left\{r_{n}\right\}_{n \geq 0}$ in Proposition 7 in such a way that $\sum_{n=0}^{\infty}\left|r_{n}(\lambda)\right|^{2}=\infty$ for each $\lambda \in C$. In this case $\sigma_{p}\left(S^{*}\right)=\varnothing$.

Corollary 8. For any Hamel basis $\left\{r_{n}\right\}_{n \geq 0}$ of $\boldsymbol{C}[z]$ there always exists another Hamel basis $\left\{q_{n}\right\}_{n \geqq 0}$ of $\boldsymbol{C}[z]$ such that

(i) $\operatorname{deg} q_{n}=n, n=0,1, \cdots$ and

(ii) the series $\sum_{n=1}^{\infty}\left|r_{n}(\lambda)\right|^{2}$ and $\sum_{n=0}^{\infty}\left|q_{n}(\lambda)\right|^{2}$ are simultaneously convergent.

Proof. Proposition 7 gives us a cyclic operators $S$ in a suitable $\mathscr{A}$. Applying the Gram-Schmidt procedure within $\mathscr{f}$ to the sequence $\left\{z^{n}\right\}_{n \geq 0}$ we get a Hamel basis $\left\{q_{n}\right\}_{n \geqq 0}$ satisfying (i). Using Proposition 6 we check that $\left\{q_{n}\right\}_{n \geqq 0}$ satisfies (ii), too.

Corollary 9. ( $\alpha$ ) For any pair of disjoint countable subsets $\tau$ and $\omega$ of $C$ there is a cyclic operator $S$ (with the normalized cyclic vector) such that $\tau \subset \sigma_{p}\left(S^{*}\right)$ and $\omega \cap \sigma_{p}\left(S^{*}\right)=\varnothing$.

( $\beta$ Let $\omega$ be a subset of $\boldsymbol{C}$ for which there are two sequences $\left\{\omega_{n}\right\}_{n \geq 1}$ and 
$\left\{\omega_{n}^{\prime}\right\}_{n \geqq 1}$ of compact set such that

$1^{\circ}$ none of $\omega_{n}$ and $\omega_{n}^{\prime}, n=1,2, \cdots$, has a bounded hole,

$2^{\circ} \quad \omega_{n} \cap \omega_{n}^{\prime}=\varnothing, n=1,2, \cdots$,

$3^{\circ} \omega=\liminf \omega_{n}$ and $C \backslash \omega=\limsup \omega_{n}^{\prime}$.

Then there is a cyclic operator $S$ such that $\sigma_{p}\left(S^{*}\right)=\omega$.

Proof. $(\alpha)$. Suppose $\tau^{*}=\left\{\lambda_{1}, \lambda_{3}, \cdots\right\}$ and $\omega^{*}=\left\{\lambda_{0}, \lambda_{2}, \cdots\right\}$. For each $n>0$ denote by $r_{n}$ the $n$-th Lagrange interpolation polynomial such that $r_{n}\left(\lambda_{k}\right)=d_{k}$ for $k=0, \cdots, n$. We can always choose the initial data $\left\{d_{k}\right\}$ in such a way that the degree of $r_{n}$ is precisely equal to $n, \sum_{n=0}^{\infty}\left|d_{2 n+1}\right|^{2}<\infty$ and $\sum_{n=0}^{\infty}\left|d_{2 n}\right|^{2}=\infty$. Setting $r_{0} \equiv 1$ we get $\sum_{n=0}^{\infty}\left|r_{n}(\lambda)\right|^{2}<\infty$ for $\lambda \in \tau^{*}$ and $\sum_{n=0}^{\infty}\left|r_{n}(\lambda)\right|^{2}=\infty$ for $\lambda \in \omega^{*}$. The sequence $\left\{r_{n}\right\}_{n \geq 0}$ satisfies all what is required in Proposition 7 .

( $\beta$ ) By $2^{\circ}$, there are open sets $\tau_{n}$ and $\tau_{n}^{\prime}$ such that $\tau_{n} \cap \tau_{n}^{\prime}=\varnothing, \omega \omega_{n}^{*} \subset \tau_{n}$ and $\omega_{n}^{\prime *} \subset \tau_{n}^{\prime}$. Let $\varphi_{n}$ be a holomorphic function defined on $\tau_{n} \cup \tau_{n}^{\prime}$ by $\varphi_{n}(z)=(2 n)^{-1}$ for $z \in \tau_{n}$ and $\varphi_{n}(z)=n+1$ for $z \in \tau_{n}^{\prime}$. The Runge theorem impliesth at there are polynomials $p_{n} \in C[z]$ such that $\left|\varphi_{n}(z)-p_{n}(z)\right| \leqq(2 n)^{-1}$ for $z \in \omega_{n}^{*} \cup \omega_{n}^{* *}$ and $\operatorname{deg} p_{n}>+\infty$ strongly. This, in turn, implies that $\left|p_{n}(z)\right| \leqq n^{-1}$ for $z \in \omega_{n}^{*}$ and $\left|p_{n}(z)\right| \geqq n$ for $z \in \omega_{n}^{\prime *}$.

If $z \in \omega^{*}=\liminf \omega_{n}^{*}$, then there is $n(z) \in N$ such that $z \in \omega_{n}^{*}$ for $n \geqq n(z)$ and, consequently, $\sum_{n=1}^{\infty}\left|p_{n}(z)\right|^{2}<+\infty$. On the other hand, if $z \in C \backslash \omega^{*}=\limsup \omega_{n}^{\prime *}$, then $z \in \omega_{n}^{\prime *}$ for infinitely many $n$ 's. Therefore, $\sum_{n=1}^{\infty}\left|p_{n}(z)\right|^{2}=+\infty$. Set $r_{n}(z)=$ $p_{m}(z)$ when $n=\operatorname{deg} \rho_{m}$ and $r_{n}(z)=z^{n} / n$ ! for $n \in \boldsymbol{N} \backslash\left\{\operatorname{deg} p_{m} ; m \geqq 1\right\}$. Since $\operatorname{deg} r_{n}=n$, the sequence $\left\{r_{n}\right\}_{n \geqq 0}$ forms a Hamel basis for $C[z]$. The resulting sequence $\left\{r_{n}\right\}_{n \geqq 0}$ has the property that $\sum_{n=0}^{\infty}\left|r_{n}(z)\right|^{2}<+\infty$ for $z \in \omega^{*}$ and $\sum_{n=0}^{\infty}\left|r_{n}(z)\right|^{2}=+\infty$ for $z \in C \backslash \omega^{*}$. A direct application of Proposition 7 completes the proof.

12. Let $\boldsymbol{r}=\left\{r_{n}\right\}_{n \geqq 0}$ be a Hamel basis for $C[z]$. Denote by $K_{r}$ the complex function defined on $\omega_{r} \times \omega_{r}$ as

$$
K_{r}(\lambda, \mu)=\sum_{n=0}^{\infty} r_{n}(\lambda)^{*} r_{n}(\mu),
$$

where $\omega_{r}=\left\{\lambda: \sum_{n=0}^{\infty}\left|r_{n}(\lambda)\right|^{2}<\infty\right\} . \quad K_{r}$ is a positive definite kernel on $\omega_{r}$. Denote by $\mathscr{H}_{\boldsymbol{r}}$ the reproducing kernel Hilbert space [2] determined by $K_{\boldsymbol{r}} \mathscr{\mathscr { P }}_{\boldsymbol{r}}$ stands for the set of all restrictions of members of $C[z]$ to $\omega_{r}$.

Theorem 6. Let $\mathscr{H}, S,\left\{r_{n}\right\}_{n \geq 0}$ and $h_{2}$ be as in Proposition 6. Suppose $\sigma_{p}\left(S^{*}\right)$ is non-empty. Then

$1^{\circ} \mathscr{P}_{\boldsymbol{r}}$ is a dense subset of $\mathscr{H}_{\boldsymbol{r}}$, 
$2^{\circ}$ the operator $M_{z}$ of multiplication by $z$ defined on $\mathscr{L}_{\boldsymbol{r}}$ is cyclic with the cyclic vector $\mathbf{1}$,

$3^{\circ}$ there is precisely one partial isometry $W: \mathscr{K}_{\rightarrow} \rightarrow \mathscr{A}_{\boldsymbol{r}}$ with the initial space clolin $\left\{h_{\lambda}: \lambda \in \sigma_{p}\left(S^{*}\right)^{*}\right\}$ and the final space $\mathscr{H}_{r}$ such that

$$
W S=M_{z} W \text {. }
$$

$4^{\circ} \quad \omega_{r}=\sigma_{p}\left(S^{*}\right)^{*}=\sigma_{p}\left(M_{z}^{*}\right)^{*}$.

Proof. Proposition 6 implies $\omega_{r}=\sigma_{p}\left(S^{*}\right)^{*}$ and

$$
\left\langle h_{\lambda}, h_{\mu}\right\rangle=K_{r}(\lambda, \mu)=\left\langle K_{r}(\lambda, \cdot), K_{r}(\mu, \cdot)\right\rangle \quad \text { for } \lambda, \mu \in \omega_{r} .
$$

This means that there is precisely one unitary operator $U$ from $\operatorname{clolin}\left\{h_{\lambda}\right.$ : $\left.\lambda \in \sigma_{p}\left(S^{*}\right)^{*}\right\}$ to $\mathscr{H}_{\boldsymbol{r}}$ such that $U h_{\lambda}=K_{\boldsymbol{r}}(\lambda, \cdot)$ for $\lambda \in \omega_{\boldsymbol{r}}$. Set $(W f)(\lambda)=\left\langle f, h_{\lambda}\right\rangle$, $f \in \mathscr{H}$ and $\lambda \in \omega_{r}$. Notice that $U h_{\lambda}=W h_{\lambda}$. Thus $U f=W f$ for $f \in \operatorname{lin}\left\{h_{\lambda}: \lambda \in \sigma_{p}\left(S^{*}\right)^{*}\right\}$. Take $f \in \operatorname{clolin}\left\{h_{\lambda}: \lambda \in \sigma_{p}\left(S^{*}\right)^{*}\right\}$. Then there is a sequence $\left\{f_{n}\right\}_{n \geqq 0} \subset$ $\operatorname{lin}\left\{h_{\lambda}: \lambda \in \sigma_{p}\left(S^{*}\right)^{*}\right\}$ such that $f_{n} \rightarrow f$. For an arbitrary $\lambda \in \omega_{r}$ we have

$$
(U f)(\lambda)=\lim _{n \rightarrow \infty}\left(U f_{n}\right)(\lambda)=\lim _{n \rightarrow \infty}\left(W f_{n}\right)(\lambda)=\lim _{n \rightarrow \infty}\left\langle f_{n}, h_{\lambda}\right\rangle=\left\langle f, h_{\lambda}\right\rangle=(W f)(\lambda) .
$$

This implies that, for $f \in \mathcal{H}, W f=U P f$, where $P$ is the orthogonal projection of $\mathscr{H}$ onto clolin $\left\{h_{\lambda}: \lambda \in \sigma_{p}\left(S^{*}\right)^{*}\right\}$. Thus $W: \mathscr{H} \rightarrow \mathscr{H}_{r}$ is a partial isometry with the initial space $\operatorname{clolin}\left\{h_{\lambda}: \lambda \in \sigma_{p}\left(S^{*}\right)^{*}\right\}$ and the final space $\mathscr{H}_{r}$. Take $p \in C[z]$. Then

$$
\left(W p(S) f_{0}\right)(\lambda)=\left\langle p(S) f_{0}, h_{\lambda}\right\rangle=p(\lambda), \quad \lambda \in \omega_{r},
$$

where $f_{0}$ is the cyclic vector of $S$. Since $S$ is cyclic, this gives us the conclusions $1^{\circ}$ and $2^{\circ}$. To prove $3^{\circ}$ take $\lambda \in \omega_{r}$ and $f=p(S) f_{0}$ with $p \in C[z]$. Then

$$
(W S f)(\lambda)=\left\langle S p(S) f_{0}, h_{\lambda}\right\rangle=\lambda p(\lambda)=\lambda(W f)(\lambda)=\left(M_{z} W f\right)(\lambda) .
$$

This establishes the conclusion $3^{\circ}$.

The only thing which remains to be proved is that $\sigma_{p}\left(S^{*}\right)^{*}=\sigma_{p}\left(M_{z}^{*}\right)^{*}$. Take $\lambda \in \sigma_{p}\left(S^{*}\right)^{*}$. Then for $\rho \in \mathscr{P}_{r}$ we have $\left\langle K_{r}(\lambda, \cdot), M_{z} p\right\rangle=\lambda^{*} p(\lambda)^{*}=$ $\lambda^{*}\left\langle K_{r}(\lambda, \cdot), p\right\rangle=\left\langle\lambda^{*} K_{r}(\lambda, \cdot), p\right\rangle$. This means that $K_{r}(\lambda, \cdot) \in \mathscr{D}\left(M_{z}^{*}\right)$ and $M_{2}^{*} K_{r}(\lambda, \cdot)=\lambda^{*} K_{r}(\lambda, \cdot)$. Consequently $\lambda \in \sigma_{p}\left(M_{z}^{*}\right)^{*}$. Conversely, suppose that $\lambda \in \sigma_{p}\left(M_{z}^{*}\right)^{*}$. This means that there is a nonzero $f \in \mathscr{D}\left(M_{z}^{*}\right)$ such that $M_{z}^{*} f=\lambda^{*} f$. Since $W$ is bounded, $3^{\circ}$ gives us $W^{*} M_{z}^{*} \subset S^{*} W^{*}$. Thus $W^{*} f \in \mathscr{D}\left(S^{*}\right)$ and $S^{*} W^{*} f=W^{*} M_{z}^{*} f=\lambda^{*} W^{*} f$. Since $W W^{*} f=f, W^{*} f$ is nonzero. This implies that $\lambda \in \sigma_{p}\left(S^{*}\right)^{*}$. This completes the proof.

Corollary 10. If $\boldsymbol{r}=\left\{r_{n}\right\}_{n \geqq 0}$ is the Hannel basis of $C[z]$, then $\mathscr{P}_{\boldsymbol{r}}$ is dense in $\mathscr{M}_{\boldsymbol{r}}$ the space $\mathfrak{M}_{\boldsymbol{r}}$ is composed of Borel measurable functions, $M_{z}$ is a cyclic operator in $\mathscr{H}_{\boldsymbol{r}}$ and $\omega_{\boldsymbol{r}}=\sigma_{p}\left(M_{z}^{*}\right)^{*}$. In particular, members of $\mathscr{A}_{\boldsymbol{r}}$ belong to the first Baire class. 
This follows from Proposition 7 and Theorem 6 .

Remark 2. Under assumptions of Theorem 6 the operator $M_{z}$ is equivalent to the operator $T$ in clolin $\left\{h_{\lambda}: \lambda \in \sigma_{p}\left(S^{*}\right)^{*}\right\}$ defined as $\mathscr{D}(T)=\operatorname{lin}\left\{P S^{n} f_{0}: n \geqq 0\right\}$ and $T P S^{n} f_{0}=P S^{n+1} f_{0}$, where $P$ is the orthogonal projection of $\mathscr{H}$ onto clolin $\left\{h_{\lambda}: \lambda \in \sigma_{p}\left(S^{*}\right)^{*}\right\}$. This suggests thinking of the operator $M_{z}$ as an analytic "model" of $S$. More precisely, we say $M_{z}$ is an analytic model of $S$ if the partial isometry appearing in Theorem 6 becomes unitary (we have to assume here tacitly that $\sigma_{p}\left(S^{*}\right)$ is nonempty). The reproducing kernel Hilbert space $\mathscr{H}_{r}$ is uniquely determined by the operator $S$ (this follows from the fact that the set of cyclic vectors is always one dimensional).

The closure $M_{z}^{-}$of $M_{z}$ is contained in the operator $M_{z}^{\max }$ defined by $\mathscr{D}\left(M_{z}^{\max }\right)=\left\{f \in \mathscr{H}_{r}: g \in \mathscr{H}_{r}, g(z)=z f(z)\right\}$ and $M_{z}^{\max } f=g$, where $g(z)=z f(z)$ (this is because the operator $M_{z}^{\max }$ is closed).

Notice finally that $\operatorname{dim} \mathscr{H}_{r}=\operatorname{card} \omega_{r}$ when $\omega_{r}$ is finite and $\operatorname{dim} \mathscr{H}_{r}=\aleph_{0}$ otherwise.

Corollary 11. Suppose $\operatorname{dim} \mathscr{H}=\infty$. A cyclic operator $S$ in $\mathscr{A}$ has an analytic model if and only if the following condition is satisfied: if $\left\{\alpha_{n}\right\}_{n \geq 0} \in l^{2}$ is such that $\sum_{n=0}^{\infty} \alpha_{n} r_{n}(\lambda)=0$, for all $\lambda \in \sigma_{p}\left(S^{*}\right)^{*}$, then $\alpha_{n}=0$ for all $n \geqq 0$.

13. Now it is the right time to enhance analytic features of the space $\mathscr{A}_{r}$. The following result will be very useful.

Lemma 3. Suppose $\operatorname{dim} \mathscr{H}=\infty$ and $S$ is a cyclic operator in $\mathscr{A}$. Let $r=\left\{r_{n}\right\}_{n \geq 0}$ be as in Proposition 6 and let $\delta>0$. Then the following conditions are equivalent:

$1^{\circ} \mathscr{A}_{\boldsymbol{r}}$ is composed of functions which are continuous on $\operatorname{disc}\left(\lambda_{0} ; \delta\right)$,

$2^{\circ} \quad \mathscr{H}_{\boldsymbol{r}}$ is composed of functions which are holomorphic on $\operatorname{disc}\left(\lambda_{0} ; \delta\right)$,

$3^{\circ} K_{r}\left(\lambda^{*}, \mu\right)$ is holomorphic in $(\lambda, \mu) \in \operatorname{disc}\left(\lambda_{0} ; \delta\right)^{*} \times \operatorname{disc}\left(\lambda_{0} ; \delta\right)$,

$4^{\circ} \quad \kappa_{s}$ is locally bounded on $\operatorname{disc}\left(\lambda_{0} ; \delta\right)$,

$5^{\circ}$ the function $\kappa_{s}$ is finite and upper semicontinuous on $\operatorname{disc}\left(\lambda_{0} ; \delta\right)$,

$6^{\circ}$ the function $\kappa_{s}$ is finite and continuous on $\operatorname{disc}\left(\lambda_{0} ; \delta\right)$,

$7^{\circ} \kappa_{s}$ is subharmonic on $\operatorname{disc}\left(\lambda_{0} ; \delta\right)$,

$8^{\circ} \int_{0}^{2 \pi} \kappa_{s}\left(\lambda_{0}+d e^{i t}\right) d t<\infty$, if $d<\delta$,

$9^{\circ} \quad \kappa_{s} \in \mathcal{L}_{10 \mathrm{c}}\left(\operatorname{disc}\left(\lambda_{0} ; \delta\right)\right)$.

If any of these conditions holds, then $\operatorname{disc}\left(\lambda_{0} ; \delta\right) \sqsubset \sigma_{p}\left(S^{*}\right)^{*}$.

Proof. $2^{\circ} \Rightarrow 3^{\circ}$. Because $K_{r}(\lambda, \cdot) \in \mathscr{K}_{r}, 2^{\circ}$ implies that $K_{r}(\lambda, \cdot)$ is holomorphic on $\operatorname{disc}\left(\lambda_{0} ; \delta\right)$ for each $\lambda \in \omega_{r}$. Since $K_{r}(\lambda, \mu)=K_{r}(\mu, \lambda)^{*}$, the function $K_{r}(\cdot, \mu)$ is antiholomorphic on $\operatorname{disc}\left(\lambda_{0} ; \delta\right)$. Hartog's theorem implies $3^{\circ}$ 
immediately.

$3^{\circ} \Rightarrow 1^{\circ}$. This follows directly from the inequality

$$
\begin{aligned}
& |f(\lambda)-f(\mu)| \leqq\|f\|\left\|K_{r}(\lambda, \cdot)-K_{r}(\mu, \cdot)\right\| \\
& =\|f\|\left(K_{r}(\lambda, \lambda)+K_{r}(\mu, \mu)-2 \operatorname{re~} K_{r}(\lambda, \mu)\right)^{1 / 2} .
\end{aligned}
$$

$1^{\circ} \Rightarrow 4^{\circ}$. Take $d<\delta$. Since any $f \in \mathscr{H}_{r}$ is continuous on $\operatorname{disc}\left(\lambda_{0} ; \delta\right)$, $\sup \left\{\left|\left\langle f, K_{r}(\lambda, \cdot)\right\rangle\right|: \lambda \in \operatorname{disc}\left(\lambda_{0} ; d\right)\right\}=\sup \left\{|f(\lambda)|: \lambda \in \operatorname{disc}\left(\lambda_{0} ; d\right)\right\}<\infty$.

An easy application of the Banach-Steinhaus theorem leads us to $\sup \left\{\kappa_{s}(\lambda) ; \lambda \in\right.$ $\left.\operatorname{disc}\left(\lambda_{0} ; d\right)\right\}=\sup \left\{\left\|K_{r}(\lambda, \cdot)\right\|^{2}: \lambda \in \operatorname{disc}\left(\lambda_{0} ; d\right)\right\}<\infty$.

$4^{\circ} \Rightarrow 2^{\circ}$. Take $f \in \mathscr{A}_{\boldsymbol{r}}$ and $d<\delta$. By Corollary 10 there is a sequence $\left\{p_{n}\right\}_{n \geqq 0} \subset \mathscr{Q}_{\boldsymbol{r}}$ such that $\left\|f-p_{n}\right\| \rightarrow 0$. Since, for $\lambda \in \operatorname{disc}\left(\lambda_{0} ; d\right)$,

$$
\mid f(\lambda)-p_{n}(\lambda) i^{2} \leqq\left\|f-p_{n}\right\| \sup \left\{\kappa_{s}(\mu): \mu \in \operatorname{disc}\left(\lambda_{0} ; d\right)\right\},
$$

$p_{n} \rightarrow f$ uniformly on $\operatorname{disc}\left(\lambda_{0} ; d\right)$. This implies $2^{\circ}$.

$3^{\circ} \Rightarrow 6^{\circ}$. Since $\kappa_{s}(\lambda)=K_{r}(\lambda, \lambda)$, the conclusion $6^{\circ}$ becomes clear.

$6^{\circ} \Leftrightarrow 5^{\circ}$. This follows directly from Corollary 6 .

$6^{\circ} \Rightarrow 7^{\circ}$. Since $\kappa_{s}(\lambda)=\lim _{k \rightarrow \infty} \sum_{n=0}^{k}\left|r_{n}(\lambda)\right|^{2}$, the function $\kappa_{s}$ is finite and continuous on $\operatorname{disc}\left(\lambda_{0} ; \delta\right)$, and, moreover, the functions $\sum_{n=0}^{k}\left|r_{n}(\lambda)\right|^{2}$ are subharmonic, we get the conclusion.

$7^{\circ} \Rightarrow 6^{\circ}$. Since $\kappa_{s}$ is finite and upper semicontinuous, applying again Corollary 6 , we get $6^{\circ}$.

$6^{\circ} \Rightarrow 8^{\circ}$ and $6^{\circ} \Rightarrow 9^{\circ}$ are obvious.

The following can be deduced from the Cauchy integral formula and from the inequality $|p(\lambda)|^{2} \leqq\|p\|^{2} \kappa_{s}(\lambda), \lambda \in C$, which holds provided $\operatorname{card} \omega_{r} \geqq \aleph_{0}$.

Sublemma 1. Suppose $\mu \in C$ and $0<d_{1}<d$. If $\int_{0}^{2 \pi} \kappa_{s}\left(\mu+d e^{\mathrm{i} t}\right) d t<\infty$ and $\lambda \in \operatorname{disc}\left(\mu ; d_{1}\right)^{-}$, then

$$
|p(\lambda)|^{2} \leqq\left(2 \pi\left(d-d_{1}\right)^{2}\right)^{-1} d^{2}\left\|\left.p\right|_{\omega_{r}}\right\|^{2} \int_{0}^{2 \pi} \kappa_{s}\left(\mu+d e^{i t}\right) d t
$$

for any $p \in C[z]$.

$8^{\circ} \Rightarrow 2^{\circ}$. Sublemma 1 guarantees that density of $\mathscr{Q}_{\boldsymbol{r}}$ in $\mathcal{H}_{r}$, which appears in Corollary 10, can be understood with respect to uniform convergence on compact subset of $\operatorname{disc}\left(\lambda_{0} ; \delta\right)$. Since members of $\mathscr{Q}_{\boldsymbol{r}}$ are holomorphic on $\operatorname{disc}\left(\lambda_{0} ; \delta\right)$, we get the conclusion.

Integrating the inequality appearing in Sublemma 1 we get

Sublemma 2. Suppose $\mu \in C, 0<d_{1}<d_{2}<d_{3}$ and $\Delta=\operatorname{disc}\left(\mu ; d_{3}\right)^{-} \backslash \operatorname{disc}\left(\mu ; d_{2}\right)^{-}$. If $\int_{\Delta} \kappa_{s}(\zeta) d m(\zeta)<\infty$ and $\lambda \in \operatorname{disc}\left(\mu ; d_{1}\right)^{-}$, then

$$
|p(\lambda)|^{2} \leqq\left(2 \pi\left(d_{2}-d_{1}\right)^{2}\left(d_{3}-d_{2}\right)\right)^{-1} d_{3}\left\|\left.p\right|_{\omega_{r}}\right\|^{2} \int_{\Delta} \kappa_{s}(\zeta) d n n(\zeta)
$$


for any $p \in C[z]$. Here $m$ denotes the (planar) Lebesgue measure.

$9^{\circ} \Rightarrow 2^{\circ}$. We can repeat the argument used in the proof of $8^{\circ} \Rightarrow 2^{\circ}$ replacing Sublemma 1 by Sublemma 2 .

We are ready to show that $\mathscr{N}_{\boldsymbol{r}}$ is composed of functions which are analytic neglecting some meager subset of int $\sigma_{p}\left(S^{*}\right)^{* 7}$.

Theorem 7. Let $S$ be a cyclic operator in $\mathscr{A}$ such that int $\sigma_{p}\left(S^{*}\right)$ is nonempty. Then

$$
\gamma(S)=\left\{\lambda \in C: \kappa_{s} \text { is finite and continuous in a neighborhood of } \lambda\right\}
$$

is an open subset of int $\sigma_{p}\left(S^{*}\right)^{*}$ such that int $\sigma_{p}\left(S^{*}\right)^{*} \backslash \gamma(S)$ is a nowhere dense subset of $\boldsymbol{C}$ (and, consequently, of int $\left.\sigma_{p}\left(S^{*}\right)^{*}\right)$. The set $\gamma(S)$ is the maximal open subset of $\omega_{\boldsymbol{r}}$ on which all functions in $\mathscr{H}_{\boldsymbol{r}}$ are holomorphic.

Proof. It is clear that $\gamma(S)$ is an open subset of int $\sigma_{p}\left(S^{*}\right)^{*}$. Due to Lemma $3\left(6^{\circ} \Rightarrow 2^{\circ}\right), \mathscr{H}_{\boldsymbol{r}}$ is composed of functions which are holomorphic on $\gamma(S)$. If all the functions in $\mathscr{H}_{r}$ are holomorphic on an open set $\gamma \subset \omega_{r}=\sigma_{p}\left(S^{*}\right)^{*}$, then, again by Lemma $3\left(2^{\circ} \Rightarrow 6^{\circ}\right), \gamma \subset \gamma(S)$.

We show that $\gamma(S)$ is dense in int $\sigma_{p}\left(S^{*}\right)^{*}$. If not, int $\sigma_{p}\left(S^{*}\right)^{*} \backslash \gamma(S)^{-}$is a nonempty open set which can be written as a union of a sequence of closed subsets $\gamma_{n}=\left\{\lambda \in \operatorname{int} \sigma_{p}\left(S^{*}\right)^{*} \backslash \gamma(S)^{-}: \kappa_{s}(\lambda) \leqq n\right\}$ of int $\sigma_{p}\left(S^{*}\right)^{*} \backslash \gamma(S)^{-}$(use Corollary 6). The Baire theorem gives us $n_{0}$ such that int $\gamma_{n_{0}}$ is nonempty. Thus there is an open disc $\Delta$ such that $\Delta$ is disjoint with $\gamma(S)$ and $\kappa_{s}$ is bounded on it. Due to Lemma $3\left(4^{\circ} \Rightarrow 6^{\circ}\right), \Delta \subset \gamma(S)$. This implies that $\gamma(S)$ is dense in int $\sigma_{p}\left(S^{*}\right)^{*}$.

Suppose that int (int $\left.\sigma_{p}\left(S^{*}\right)^{*} \backslash \gamma(S)\right)^{-}$is nonempty. Since (int $\left.\sigma_{p}\left(S^{*}\right)^{*} \backslash \gamma(S)\right)^{-}$ $\subset$ (int $\left.\sigma_{p}\left(S^{*}\right)^{*}\right)^{-} \backslash \gamma(S)$, we check that there is an open disc contained in int $\sigma_{p}\left(S^{*}\right)^{*} \backslash \gamma(S)^{-}$. This would mean that $\gamma(S)$ was not dense in int $\sigma_{p}\left(S^{*}\right)^{*}$. This contradicts what we have proved so far.

Example 2. In order to illustrate Theorem 7 we propose considering the closed annulus $\omega=\operatorname{disc}(0 ; 2)^{-} \backslash \operatorname{disc}(0 ; 1)$. One can construct, using the Runge theorem, a sequence $\left\{r_{k_{n}}\right\}_{n \geqq 0}$ of polynomials from $C[z]$ such that

(25) $k_{n}=\operatorname{deg} r_{k_{n}}$ strongly increases,

(26) $\sum_{n=0}^{\infty}\left|r_{k_{n}}(\lambda)\right|^{2}=\infty$ for $\lambda \in C \backslash \omega$,

(27) $\sum_{n=0}^{\infty}\left|r_{k_{n}}(\cdot)\right|^{2}$ is uniformly convergent on compact sets of the form $\{t \exp (i \theta)$ : $1 \leqq t \leqq 2, \varepsilon \leqq \theta \leqq 2 \pi\}, \varepsilon>0$,

\footnotetext{
${ }^{7}$ For bounded subnormal operators a similar result has been proved in $[20$, Th. 1.2$]$.
} 
(28) $\left|r_{k_{n}}\left(t \exp \left[(n+1)^{-1} \mathrm{i}\right]\right)\right| \geqq n$ for $1 \leqq t \leqq 2$ and all $n$.

Put $r_{n}(z)=\frac{z^{n}}{n !}$ for $n \in N \backslash\left\{k_{m}: m \geqq 0\right\}$. Then the extended sequence $\left\{r_{n}\right\}_{n \geqq 0}$ shares the properties (26) and (27) with the initial $\left\{r_{k_{n}}\right\}_{n \geqq 0}$. Since $\operatorname{deg} r_{n}=n$ (cf. (25)), the sequence $\left\{r_{n}\right\}_{n \geqq 0}$ forms a Hamel basis for $\boldsymbol{C}[z]$. Due to Proposition 7, there exists a cyclic operator $S$ such that $\sigma_{p}\left(S^{*}\right)^{*}=$ $\left\{\lambda \in C: \sum_{n=0}^{\infty}\left|r_{n}(\lambda)\right|^{2}<\infty\right\}$ and $\kappa_{s}(\lambda)=\sum_{n=0}^{\infty}\left|r_{n}(\lambda)\right|^{2}$. It can be deduced directly from our construction that $\kappa_{s}$ is finite and continuous in int $\omega \backslash(1,2)$. Moreover, the evaluation (28) implies $\kappa_{s}$ is not continuous at any point of the interval $(1,2)$ (though its restriction to $(1,2)$ is finite and continuous). So $\gamma(S)=\operatorname{int} \omega \backslash(1,2) \neq$ int $\sigma_{p}\left(S^{*}\right)^{*}$.

As Example 2 shows, it may happen that $\sigma_{p}\left(S^{*}\right)$ has bounded holes. This is related to the lack of full analyticity of the functional model we have considered so far. The following result sheds more light on this question.

Theorem 8. Let $S$ be a cyclic operator in $\mathcal{H}$. Then $\gamma(S)$ has the following property: if $\omega$ is a bounded open set in $C$ such that either $\kappa_{s}$ is bounded on $\partial \omega$ or $\partial \omega \subset \gamma(S)$, then $\omega \subset \gamma(S)$.

Proof. Suppose $\kappa_{s}$ is bounded on $\partial \omega$. Then Corollary 6 implies that $\kappa_{s}$ is bounded on $\omega$. Due to Lemma $3\left(4^{\circ} \Rightarrow 6^{\circ}\right)$, we have $\omega \subset \gamma(S)$.

Suppose $\partial \omega \subset \gamma(S)$. Then Lemma 3 guarantees that $\kappa_{s}$ is finite and continuous on $\gamma(S)$. Since $\partial \omega$ is compact, $\kappa_{s}$ is bounded on $\partial \omega$. The previous paragraph yields the conclusion.

Proposition 8. Suppose $\operatorname{dim} \mathscr{H}=\infty$ and $S$ is a cyclic operator in $\mathscr{H}$. If $f \in \mathscr{D}\left(S^{-}\right), S^{-} f=\lambda f$, either $\lambda \in C \backslash \sigma_{p}\left(S^{*}\right)^{*}$ or $\lambda \in \gamma(S)$, then $f$ is orthogonal to clolin $\left\{h_{\mu}: \mu \in \sigma_{p}\left(S^{*}\right)^{*}\right\}$, where $h_{\mu}$ is as in Proposition 6 .

Proof. Theorem 6 says that $W S=M_{z} W, W$ is a partial isometry. Since $W$ is bounded, $W S^{-} \subset M_{z}^{-} W$. Asumme $S^{-} f=\lambda f, f \in \mathscr{D}\left(S^{-}\right)$. Then $W f \in \mathscr{D}\left(M_{z}^{-}\right)$ and $M_{z}^{-} W f=\lambda W f$. Take $\lambda$ which is not in $\omega_{r}=\sigma_{p}\left(S^{*}\right)^{*}$. Since $(\lambda-z)(W f)(z)=0$, for $z \in \omega_{r}$, we have $W f=0$. Take now $\lambda \in \gamma(S)$. Since $(\lambda-z)(W f)(z)=0$, for $z \in \omega_{r}$, we get $W f=0$ on $\omega_{r} \backslash\{\lambda\}$. Due to Lemma $3, W f$ is analytic on $\gamma(S)$. Since $W f=0$ on $\gamma(S) \backslash\{\lambda\}$ and $\lambda \in \gamma(S)$, we have $(W f)(\lambda)=0$. Because $W f=0$ on $\omega_{r} \backslash\{\lambda\}$, we get $W f=0$. The conclusion follows from $W f=W P f$, where $P$ is the orthogonal projection of $\mathscr{H}$ onto clolin $\left\{h_{\mu}: \mu \in \sigma_{p}\left(S^{*}\right)^{*}\right\}$.

Corollary 12. Suppose $\operatorname{dim} \mathscr{H}=\infty$ and $S$ is a cyclic operator in $\mathscr{A}$. If $S$ has an analytic model ( $c f$. Remark 2), then $S$ is closable and $1^{\circ} \sigma_{p}\left(S^{-}\right) \subset \sigma_{p}\left(S^{*}\right)^{*} \backslash \gamma(S)$, 


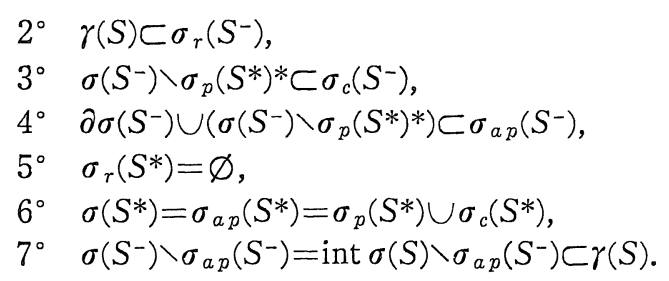

Proof. Since $S$ is unitarily equivalent to $M_{z}$ and $M_{z}$ is closable (cf. Remark 2), $S$ is closable, too.

Proposition 8 implies directly $1^{\circ}$. Since always

$$
\sigma\left(S^{-}\right) \backslash \sigma_{p}\left(S^{*}\right)^{*} \subset \sigma_{p}\left(S^{-}\right) \cup \sigma_{c}\left(S^{-}\right),
$$

the conclusion $3^{\circ}$ follows from Proposition 8. Using again Proposition 8 and $\sigma_{p}\left(S^{*}\right)^{*} \subset \sigma_{p}\left(S^{-}\right) \cup \sigma_{r}\left(S^{-}\right)$, we get the conclusion $2^{\circ}$. As $\sigma_{c}(A) \subset \sigma_{a p}(A)$ for a closed $A$, the conclusion $4^{\circ}$ follows from $3^{\circ}$ and (5). Emptiness of $\sigma_{r}\left(S^{*}\right)$ can be easily deduced from $1^{\circ}$. This, in turn, implies $6^{\circ}$.

The conclusion $7^{\circ}$ follows from what is in the proof of Theorem 9 .

14. Now we come back to subnormal operators. Our goal here is to find relationship between functional and analytic models (cf. Remark 2) for such operators.

Consider a cyclic subnormal operator $S$ in $\mathscr{K}(\operatorname{dim} \mathscr{K}=\infty)$ with the cyclic vector $f_{0}$. Let $\mathfrak{m}$ be a representing measure of $S$ (cf. Section 9 ). Then there is a unique unitary operator $V_{\mathfrak{m}}: \mathscr{H}_{\mathfrak{m}} \rightarrow \mathscr{H}$ such that $V_{\mathfrak{m}} p=p(S) f_{0}, p$ is an analytic polynomial in $\mathcal{H}_{\mathfrak{m}}$. Denote by $U_{\mathfrak{m}}$ the unitary operator $W V_{\mathfrak{m}}: \mathscr{H}_{\mathfrak{m}} \rightarrow \mathscr{H}_{r}$, provided $\sigma_{p}\left(S^{*}\right)$ is nonempty. More explicitly, $\left(U_{\mathfrak{m}} p\right)(\lambda)=p(\lambda), \lambda \in \sigma_{p}\left(S^{*}\right)^{*}$. In general, $p$ can not be replaced by other functions. However, we are able to determine those $\lambda$ 's for which the equality

$$
\left(U_{\mathfrak{m}} f\right)(\lambda)=f(\lambda)
$$

holds for all $f$ in $\mathscr{H}_{\mathfrak{m}}$.

Proposition 9. For each $f \in \mathcal{H}_{\mathfrak{m}}$, equality (29) holds for $\mathfrak{m}$-almost all $\lambda$ in $\sigma_{p}\left(S^{*}\right)^{*} \cap \operatorname{supp} \mathfrak{m}$. In particular, $U_{\mathfrak{m}} f$ is holomorphic on $\operatorname{int}(\gamma(S) \cap \operatorname{supp} \mathfrak{m})$.

Proof. If $f \in \mathscr{H}_{\mathrm{m}}$, then there is a sequence $\left\{p_{n}\right\}_{n \geq 0} \subset C[z]$ such that $\int\left|f-p_{n}\right|^{2} d \mathfrak{m} \rightarrow 0$ as $n \rightarrow \infty$. Since $\left(U_{\mathrm{m}} p_{n}\right)(\lambda)=p_{n}(\lambda)$ for $\lambda \in \sigma_{p}\left(S^{*}\right)^{*} \cap \operatorname{supp} \mathfrak{m}$, we get $\left(U_{\mathfrak{m}} f\right)(\lambda)=\lim \left(U_{\mathfrak{m}} p_{n}\right)(\lambda)=\lim p_{n}(\lambda)$ for $\lambda \in \sigma_{p}\left(S^{*}\right)^{*} \cap$ supp m. Passing to a subsequence if necessary, we can assume that $p_{n} \rightarrow f \mathfrak{m}$-almost everywhere. This gives us the first part of the conclusion. The other follows from Lemma 3 . 
It may happen, as the example of the classical shift in the Hardy space of the unit disc shows, that $\sigma_{p}\left(S^{*}\right)^{*} \cap \operatorname{supp} \mathfrak{m}=\varnothing$; also other cases are possiblecf. Section 16. In general, the goal would be to make int $\sigma_{p}\left(S^{*}\right)^{*} \cap \operatorname{supp}$ m as large as possible. The following result goes in this direction.

Proposition 10. Let $S$ be a cyclic subnormal operator in $\mathscr{A}$ and let $N$ be its minimal normal extension of spectral type. If $\left\{\omega_{n}\right\}_{n \geqq 0}$ is a collection of bounded holes of $\sigma(N)$ with the following property:

(30) for every $n$ there is a bounded open set $\tau_{n}$ such that $\omega_{n}^{-} \subset \tau_{n}$ and $\partial \tau_{n} \subset \gamma(S)$, then for an arbitrary $\varepsilon>0$ there exists a cyclic subnormal operator $R$ in $\mathscr{H}$ and a positive operator $X$ in $\mathscr{H}$ such that

$1^{\circ} \quad R$ has a minimal normal extension $M$ of spectral type for which

$$
\sigma(M)=\bigcup_{n} \omega_{n} \cup \sigma(N),
$$

$2^{\circ} X$ establishes similarity between $R$ and $S$, i.e. $R=X S X^{-1}$,

$3^{\circ} \quad\|f\|^{2} \leqq\|X f\|^{2} \leqq(1+\varepsilon)\|f\|^{2}$ for $f \in \mathcal{H}$.

Proof. Consider the functional model for $S$ given by $\mathfrak{m}=\mathfrak{m}_{N}$. Owing Theorem 5, $\sigma(N)=\operatorname{supp} \mathfrak{m}$. Set $\mathfrak{n}=\mathfrak{m}+\sum_{n} c_{n} m_{n}$, where $m_{n}$ is the restriction of the planar Lebesque measure to $\omega_{n}$,

$$
c_{n}=\varepsilon_{n}\left(\max \left\{1, \sup \left\{|z| ; z \in \omega_{n}\right\}\right\}\right)^{-n}\left(m_{n}\left(\omega_{n}\right) \max \left\{1, \sup \left\{\kappa_{s}(\lambda) ; \lambda \in \partial \tau_{n}\right\}\right\}\right)^{-1}
$$

and $\sum_{n=0}^{\infty} \varepsilon_{n}=\varepsilon$ with $\varepsilon_{n}>0$ (by Lemma $3, \sup \left\{\kappa_{s}(\lambda) ; \lambda \in \partial \tau_{n}\right\}<\infty$ ). One can check that such a measure $\mathfrak{n}$ satisfies (13) and $\operatorname{supp} \mathfrak{n}=\bigcup_{n} \omega_{n} \cap \sigma(N)$. Consider the cyclic subnormal operator $S_{\mathfrak{n}}$ (cf. Section 8) and its minimal normal extension $N_{\mathfrak{n}}$. Owing conclusion $5^{\circ}$ of Theorem $5, \sigma\left(N_{\mathfrak{n}}^{\prime}\right)=\bigcup_{n} \tau_{n} \cup \sigma(N)$.

We show that $S_{\mathfrak{n}}$ is similar to $S_{\mathfrak{m}}$. Define $Y f=g$, where $g$ is the restriction of $f \in \mathscr{K}_{\mathfrak{n}}$ to suppm. The operator $y$ establishes similarity between $\mathscr{K}_{\mathfrak{n}}$ and $\mathscr{H}_{\mathfrak{m}}$. To see this check that

$$
\|Y f\|_{\mathfrak{m}} \leqq\|f\|_{\mathfrak{n}}
$$

and, consequently, $Y \mathscr{H}_{\mathrm{n}} \subset \mathscr{H}_{\mathrm{n}}$. Since the polynomials are dense in both $\mathscr{H}_{\mathrm{n}}$ and $\mathscr{H}_{\mathfrak{m}}, Y \mathscr{H}_{\mathfrak{n}}$ is dense in $\mathscr{H}_{\mathrm{r}}$.

$$
\|p\|_{\mathfrak{n}}^{2} \leqq(1+\varepsilon)\|y p\|_{\mathfrak{m}}^{2}, \quad p \in \boldsymbol{C}[z] .
$$

The maximum modulus principle gives us, for $\lambda \in \tau_{n}$,

$$
|p(\lambda)|^{2} \leqq \sup \left\{\kappa_{s}(\mu) ; \mu \in \partial \tau_{n}\right\}\|p\|_{\text {iil }}^{2}, \quad p \in C[z] .
$$

This implies 


$$
\|p\|_{\mathfrak{n}}^{2} \leqq\|p\|_{\mathfrak{m}}^{2}+\sum_{n \geq 0} c_{n} m_{n}\left(\omega_{n}\right) \sup \left\{\kappa_{s}(\lambda) ; \lambda \in \partial \tau_{n}\right\}\|p\|_{\mathfrak{m}}^{2} \leqq(1+\varepsilon)\|p\|_{\mathfrak{m}}^{2} .
$$

The inequalities (31) and (32) force both $Y$ and $Y^{-1}$ to be bounded. It is evident that $S_{\mathrm{m}} Y=Y S_{\mathrm{n}}$. The polar decomposition of $Y^{-1}, Y^{-1}=V\left|Y^{-1}\right|$, provides us with a unitary operator $V$ and a positive similarity $X=\left|Y^{-1}\right|$ in $\mathscr{H}_{\mathrm{m}}$ such that $R X=X S_{\mathfrak{m}}$ where $R=V^{-1} S_{\mathfrak{n}} V$. The remaining parts of the conclusion follows from (31) and (32).

Theorem 9. Let $S$ be a cyclic subnormal operator in $\mathscr{K}$, which has an analytic model. Then $\gamma(S)=\sigma\left(S^{-}\right) \backslash \sigma_{a p}\left(S^{-}\right)=\operatorname{int} \sigma\left(S^{-}\right) \backslash \sigma_{a p}\left(S^{-}\right)$. In particular, if $\gamma(S)=C$, then $\sigma_{a p}\left(S^{-}\right)=\varnothing .^{8}$

Proof. Let $\mathfrak{m}$ be a representing measure of $S$. Without loss of generality we may assume (cf. Theorem 5) that $S=S_{\mathfrak{m}}$. First we show that $\gamma(S) \subset \sigma\left(S^{-}\right) \backslash$ $\sigma_{a p}\left(S^{-}\right)$. Take $\lambda_{0} \in \gamma\left(S_{\mathfrak{m}}\right)$. Since $\gamma\left(S_{\mathfrak{m}}\right) \subset \sigma\left(S_{\mathfrak{m}}^{*}\right)^{*}=\sigma\left(S_{\mathrm{m}}^{-}\right)$, we have $\lambda_{0} \in \sigma\left(S_{\mathrm{m}}^{-}\right)$. It is enough to show that $\lambda_{0}$ is not in $\sigma_{a p}\left(S_{\mathrm{m}}^{-}\right)$. Consider $\varepsilon>0$ such that $\operatorname{disc}\left(\lambda_{0} ; \varepsilon\right)^{-} \subset \gamma\left(S_{\mathfrak{m}}\right)$. By Lemma $3, \quad c=\left(\sup \left\{\kappa_{s}(\lambda) ; \lambda \in \operatorname{disc}\left(\lambda_{0} ; \varepsilon\right)^{-}\right\}\right)^{1 / 2}<\infty$. Pick $p \in C[z]$. Applying the maximum modulus principle to the holomorphic function $p$, we get, by Proposition $6\left(2^{\circ}\right)$, that, for $\lambda \in \operatorname{disc}\left(\lambda_{0} ; \varepsilon\right)^{-}$,

$$
|p(\lambda)| \leqq c \varepsilon^{-1}\left\|\left(\lambda_{0}-S_{\mathfrak{m}}\right) p\right\|_{\mathfrak{m}} .
$$

Using (33) we have (with $\left.\Delta=\operatorname{disc}\left(\lambda_{0} ; \varepsilon\right)^{-}\right)$

$$
\begin{aligned}
& \quad\|p\|_{\mathfrak{m}}^{2}=\int_{\Delta}|p|^{2} d \mathfrak{m}=\int_{C \backslash \Delta}|p|^{2} d \mathfrak{m} \leqq \sup \left\{|p(\lambda)|^{2} ; \lambda \in \Delta\right\} \mathfrak{m}(\Delta) \\
& +\varepsilon^{-2} \int_{C \backslash \Delta}\left|\left(\lambda_{0}-S_{\mathfrak{m}}\right) p\right|^{2} d \mathfrak{m} \leqq \varepsilon^{-2}\left(c^{2} \mathfrak{m}(\Delta)+1\right)\left\|\left(\lambda_{0}-S_{\mathfrak{m}}\right) \rho\right\|_{\mathfrak{m}}^{2} .
\end{aligned}
$$

This implies that $\lambda_{0}-S^{-}$is bounded from bellow. Consequently $\lambda_{0} \in \sigma\left(S^{-}\right) \backslash$ $\sigma_{a p}\left(S^{-}\right)$.

To prove the reverse inclusion ${ }^{9}$ we need the following result (which can be proved in the same way as in the bounded case; cf., [8, p. 172]):

Suppose we are given a closed operator $A$ in $\mathscr{H}$ and $\lambda_{0} \in \sigma(A) \backslash \sigma_{a p}(A)$. Then there is an open neighborhood $\omega$ of $\lambda_{0}$ and a holomorphic function $\varphi: \omega \rightarrow \mathscr{H}$ such that $\varphi(\lambda) \neq 0$ and $\varphi(\lambda) \in \operatorname{ker}(A-\lambda)^{*}$ for $\lambda \in \omega$.

Take $\lambda_{0} \in \sigma\left(S^{-}\right) \backslash \sigma_{a p}\left(S^{-}\right)$. Then there is the function $\varphi$ having the properties just described. Since $\varphi(\lambda) \in \operatorname{ker}\left(S^{*}-\lambda^{*}\right)$ and $\varphi(\lambda) \neq 0$, we have $\lambda \in \sigma_{p}\left(S^{*}\right)^{*}$. From the proof of the uniqueness part of Lemma 2, we infer that $h_{\lambda}=$ $\left\langle h_{\lambda}, f_{0}\right\rangle\left\langle\varphi(\lambda), f_{0}\right\rangle^{-1} \varphi(\lambda)=\left\langle\varphi(\lambda), f_{0}\right\rangle^{-1} \varphi(\lambda)$ for $\lambda \in \omega$. Since $\varphi$ is holomorphic, $\left\|h_{\lambda}\right\|$ is locally bounded on $\omega$. Lemma $3,4^{\circ}$ implies that $\lambda_{0} \in \gamma(S)$.

\footnotetext{
${ }^{8}$ For bounded operators a similar result has been proved in [20, Th. 1.1].

9 This inclusion is, as the proof shows, true for an arbitrary cyclic operator.
} 
An application of (5) gives us

$$
\sigma\left(S^{-}\right) \backslash \sigma_{a p}\left(S^{-}\right)=\operatorname{int} \sigma\left(S^{-}\right) \backslash \sigma_{a p}\left(S^{-}\right) .
$$

This completes the proof.

Corollary 13. Suppose $S$ is as in Theorem 9. Then $S$ has, in addition to the properties described in Corollary 12, the following ones:

$$
\begin{gathered}
\partial \sigma\left(S^{-}\right) \subset \sigma_{a p}\left(S^{-}\right)=\sigma\left(S^{-}\right) \backslash\left(\gamma(S) \underset{n \geqq 0}{\bigcup} \omega_{n}\right), \\
\sigma_{p}\left(S^{-}\right) \leqq \sigma_{p}\left(S^{*}\right)^{*} \backslash\left(\gamma(S) \underset{n \geqq 0}{\bigcup} \omega_{n}\right),
\end{gathered}
$$

where $\left\{\omega_{n}\right\}_{n \geqq 0}$ is the collection of all the holes of $\sigma(N), N$ being a minimal normal extension of $S$ of spectral type.

This follows from Theorem 9 and Corollary 12 (and the fact that $\left.\omega_{n} \cap \sigma_{a p}\left(S^{-}\right)=\varnothing\right)$.

A natural question which appears here is to describe circumstances under which a hole of $\sigma(N)$ is contained in $\sigma_{p}\left(S^{*}\right)^{*}$. Some answer to this question for bounded operators has been given in [21].

The result which follows provides us with additional information about cyclic subnormal operators having analytic models.

Proposition 11. Let $M_{z}$ acting in $\mathscr{H}_{\boldsymbol{r}}$ be an analytic model of a cyclic subnormal operator. If int $\sigma_{p}\left(M_{z}^{*}\right)$ is nonempty, then $\mathscr{Q}_{r}$ is a core for $M_{z}^{\max }$ that is $M_{z}^{\max }=M_{z}^{-}$.

Proof. Since int $\sigma_{p}\left(M_{z}^{*}\right)$ is nonempty, the same is true for $\gamma\left(M_{z}\right)$, due to Theorem 7. Take $\lambda_{0} \in \gamma\left(M_{z}\right)$. Then, by Theorem $9, \lambda_{0}$ is not in $\sigma_{a p}\left(M_{z}^{-}\right)$. If $f$ is in $\mathscr{D}\left(M_{z}^{\max }\right)$, then $\left(\lambda_{0}-M_{z}^{\max }\right) f \in \mathscr{H}_{r}$. Thus there is a sequence of polynomials $\left\{q_{n}\right\}_{n \geq 0} \subset \mathscr{P}_{r}$ such that $q_{n} \rightarrow\left(\lambda_{0}-M_{z}^{\max }\right) f$. However $q_{n}\left(\lambda_{0}\right) \rightarrow 0$. Consequently, $t_{n}=q_{n}-q_{n}\left(\lambda_{0}\right) \in \mathscr{P}_{r}, t_{n} \rightarrow\left(\lambda_{0}-M_{z}^{\max }\right) f$ and $t_{n}$ vanish at $\lambda_{0}$. This implies that there exist polynomials $p_{n} \in \mathscr{P}_{r}$ such that $t_{n}=\left(\lambda_{0}-M_{z}^{-}\right) p_{n}$. Thus $\left(\lambda_{0}-M_{z}^{-}\right)^{-1} t_{n}=p_{n}$. Since $\left(\lambda_{0}-M_{z}^{-}\right)^{-1}$ is continuous, $\rho_{n}$ approaches some $f_{1} \in \mathcal{K}_{\boldsymbol{r}}$. However, $\quad\left(\lambda_{0}-M_{z}^{-}\right) p_{n}=t_{n} \rightarrow\left(\lambda_{0}-M_{z}^{\max }\right) f$. Closedness of $\lambda_{0}-M_{z}^{-}$implies $\left(\lambda_{0}-M_{z}^{\max }\right) f=\left(\lambda_{0}-M_{z}^{-}\right) f_{1}$. This, in turn, implies $f=f_{1}$ exept $\lambda_{0}$. Since both these functions are analytic in a neighbourhood of $\lambda_{0}$ (cf. Lemma 3), they are equal. Finally, we get $p_{n} \rightarrow f$ and $M_{z}^{-} p_{n}=\lambda_{0} p_{n}-\left(\lambda_{0}-M_{z}^{-}\right) p_{n} \rightarrow \lambda_{0} f-\left(\lambda_{0}-M_{z}^{\max }\right) f$ $=M_{z}^{\max } f$. This completes the proof.

Remark 3. The functional model we have presented is determined by a particular measure satisfying (13) and (14) and the reproducing kernel property (18) can be satisfactorily described by means of the measure in question. This allows to modify the measure still preserving the crucial property (18). Here 
is a sample:

Let $\mathfrak{m}$ be a positive measure on $C$ satisfying (13). If int $\sigma_{p}\left(S_{\mathrm{w}}^{*}\right)$ is nonempty then $\sigma_{p}\left(S_{\mathfrak{n}}^{*}\right) \subset \sigma_{p}\left(S_{\mathfrak{n}}^{*}\right)$ and $\gamma\left(S_{\mathfrak{m}}\right) \subset \gamma\left(S_{\mathfrak{n}}\right)$, where $\mathfrak{n}=\left(1+|z|^{2}\right) \mathfrak{m}$.

The inclusion $\sigma_{p}\left(S_{\mathfrak{n}}^{*}\right) \subset \sigma_{p}\left(S_{\mathfrak{n}}^{*}\right)$ follows from Proposition 6 and the inclusion $\gamma\left(S_{\mathfrak{n}}\right) \subset \gamma\left(S_{\mathfrak{n}}\right)$ follows from Lemma 3.

The inclusion $5^{\circ}$ of Theorem 1 can be proved for cyclic $S$ directly using the property (18). Indeed, if $\lambda \in \sigma_{p}(N)$, then for every $p \in \mathbb{C}[z]$ we have $|p(\lambda)|^{2}$ $\leqq \mathfrak{m}^{\prime}(\{\lambda\})^{-1}\|p\|_{\mathfrak{m}}^{2}$, where $\mathfrak{m}=\mathfrak{m}_{N}$. Lemma 2 completes the argument.

\section{All This for Weighted Shifts}

15. Consider at the beginning a cyclic operator $S$ with the cyclic vector $f_{0}$, which satisfies the following condition (cf. [18, Lemma 1])

$$
\left\langle S^{m} f_{0}, S^{n} f_{0}\right\rangle=a_{m+n} b_{m-n}, \quad m, n \geqq 0,
$$

where $\left\{a_{k}\right\}_{k \geqq 0}$ is a Hamburger moment sequence and $\left\{b_{k}\right\}_{k=-\infty}^{+\infty}$ is a trigonometric moment sequence.

If $b_{k}=\delta_{k}$, the usual "zero-one" sequence supported at zero, then $S$ becomes a weighted shift operator (the traditional definition will be given later). In this case the sequence $\left\{a_{n}\right\}_{n \geqq 0}$ can be always replaced [18, Theorem 4] by the Stieltjes moment sequence $\left\{c_{k}\right\}_{n \geqq 0}$ defined as $c_{k}=\int_{0}^{+\infty} x^{k} d \mathfrak{a}^{\prime}(x), k \geqq 0$, where $\mathfrak{a}^{\prime}$ is determined by $a_{2 k}=\int_{0}^{+\infty} x^{2 k} d \mathfrak{a}^{\prime}(x), k \geqq 0$ (such a measure $\mathfrak{a}^{\prime}$ always exists because $\left\{a_{2 k}\right\}_{k \geq 0}$ is a Stieltjes moment sequence!).

Relating the operator $S$ to some weighted shift we localize the set on which it may have an analytic model.

Theorem 10. Suppose $\operatorname{dim} \mathscr{H}=\infty$. Let $S$ be a cyclic operator satisfying (34) with $\left\{a_{k}\right\}_{k \geqq 0}$ being a Hamburgdr moment sequence and with $\left\{b_{k}\right\}_{k=-\infty}^{+\infty}$ such that there is $c>0$ for which

$$
\sum_{m, n \geq 0} b_{m-n} \zeta_{m} \zeta_{n}^{*} \geqq c \sum_{n \geqq 0}\left|\zeta_{n}\right|^{2}
$$

where $\zeta_{0}, \cdots, \zeta_{k}$ is an arbitrary finite sequence of complex numbers. If $S$ is bounded, then

Otherwise

$$
\gamma(S)=\operatorname{disc}(0 ;\|S\|) \subset \sigma_{p}\left(S^{*}\right)^{*} \subset \operatorname{disc}(0 ;\|S\|)^{-},
$$

$$
\gamma(S)=\sigma_{p}\left(S^{*}\right)^{*}=C
$$

Proof. Denote by $\mathfrak{a}$ and $\mathfrak{b}$ representing measures of the moment sequences $\left\{a_{k}\right\}_{k \geq 0}$ and $\left\{b_{k}\right\}_{k=-\infty}^{+\infty}$ respectively. Take $\zeta_{0}, \cdots, \zeta_{k} \in C$. Then, the inequality (35) can be written by means of $\mathfrak{b}$ as 


$$
\int_{0}^{2 \pi}\left|\sum_{n=0}^{k} \zeta_{n} e^{\mathrm{i} n t}\right|^{2} d \mathfrak{b}(t) \geqq c(2 \pi)^{-1} \int_{0}^{2 \pi}\left|\sum_{n=0}^{k} \zeta_{n} e^{\mathrm{i} n t}\right|^{2} d t
$$

Since trigonometric polynomials $\sum_{n=-k}^{k} \zeta_{n} e^{\mathrm{i} n t}$ are dense in $\mathcal{L}^{2}(\mathfrak{b})$, the above inequality implies

$$
\int_{0}^{2 \pi} f d\left(\mathfrak{b}-c(2 \pi)^{-1} m\right) \geqq 0 \quad \text { for all nonnegative } f \text { in } \mathcal{L}^{1}(\mathfrak{b})
$$

and, consequently, the measure $\mathfrak{b}-c(2 \pi)^{-1} m$ is nonnegative. Define the measure m on $C$ as

$$
\mathfrak{m}(\sigma)=\int_{0}^{2 \pi} \int_{-\infty}^{+\infty} \chi_{\sigma}\left(r e^{\mathrm{i} t}\right) d \mathfrak{a}(r) d \mathfrak{b}(t) \quad \text { for Borel } \sigma
$$

(here $\chi_{\sigma}$ stands for the characteristic (indicator) function of $\sigma$ ). It easy to see that $\mathfrak{m}$ is a representing measure of $S$ (use (34)). Moreover, its support is contained in $\operatorname{disc}(0 ; a)^{-}, a=\lim \left(a_{2 n}\right)^{1 / 2 n}$, (with convention: $\operatorname{disc}(0 ;+\infty)=C$ ). Indeed, it is known that

$$
a=\sup \{|t| ; t \in \operatorname{supp} a\} .
$$

Pick a complex number $\lambda$ with $|\lambda|>a$. Then there is an open neighbourhood $\omega$ of $\lambda$ such that $r \in \boldsymbol{R}, t \in[0,2 \pi)$ and $r e^{i t} \in \omega$ imply $|r|>a$ and consequently $\chi_{\omega}\left(r e^{\mathrm{i} t}\right)=0$. This and (37) force

$$
\int_{-\infty}^{+\infty} \chi_{\sigma}\left(r e^{i t}\right) d \mathfrak{a}(r)=0 \quad \text { for all } t
$$

This, in turn, means $\mathfrak{m}(\omega)=0$. Thus $\lambda$ is not in supp $\mathfrak{m}$.

Since $\left\{a_{2 k}\right\}_{k \geqq 0}$ is a Stieltjes moment sequence, there is a positive measure $\mathfrak{a}^{\prime}$ on $[0,+\infty)$ such that

$$
a_{2 k}=\int_{0}^{+\infty} x^{2 k} d \mathfrak{a}^{\prime}(x), \quad k \geqq 0 .
$$

There is a weighted shift $S_{a^{\prime}}$ in $\mathscr{H}$ such that

$$
\left\langle S_{\mathfrak{a}^{\prime}}^{m} f_{0} S_{\mathfrak{u}^{\prime}}^{n} f_{0}\right\rangle=\int_{0}^{+\infty} x^{m+n} d \mathfrak{a}^{\prime}(x) \delta_{m-n}, \quad m, n \geqq 0 .
$$

Take $\rho \in C[z]$. Then, by (36), (38) and (39), we have

$$
\begin{aligned}
& \left\|\rho(S) f_{0}\right\|^{2}=\int|\rho|^{2} d \mathfrak{m}=\int_{-\infty}^{+\infty} \int_{0}^{2 \pi}\left|\rho\left(r e^{\mathrm{i} t}\right)\right|^{2} d \mathfrak{b}(t) d \mathfrak{a}(r) \\
& \geqq c(2 \pi)^{-1} \int_{-\infty}^{+\infty} \int_{0}^{2 \pi}\left|\rho\left(r e^{\mathrm{i} t}\right)\right|^{2} d t d \mathfrak{a}(r)=c(2 \pi)^{-1} \int_{-\infty}^{+\infty} \int_{0}^{2 \pi}\left|\rho\left(r e^{\mathrm{i} t}\right)\right|^{2} d t d \mathfrak{a}^{\prime}(r) \\
& =c\left\|\rho\left(S_{\mathfrak{a}^{\prime}}\right) f_{0}\right\|^{2} .
\end{aligned}
$$

Notice $^{10}$ that

${ }^{10}$ Such a situation appears in [9] and [10] where, unfortunately, the case when the representing measure of the Stieljes moment sequence $\left\{a_{2 n}\right\}$ (in our notation) has zero as its atom is excluded. This is an unnecessary restricton which comes from Theorem 8 of [9]. However this theorem does not tell the whole truth; it may happen that zero is an atom of a representing measure of a subnormal weighted shift (consider the moment sequence $a_{0}=1, a_{2 n}=(2 n+2)^{-1}$ for $\left.n>0\right)$ 


$$
\operatorname{disc}(0 ; a) \subset \gamma^{\prime}\left(S_{a^{\prime}}\right) .
$$

To prove this consider first the case $a \in \operatorname{supp} \mathfrak{a}^{\prime}$. Then, applying the Cauchy integral formula to the circle $(0 ; a)$ and the Schwarz inequality we get $\kappa_{s_{a^{\prime}}}(\lambda) \leqq$ $a^{2}(a-|\lambda|)^{-2} a^{\prime}(\{a\})^{-1}$ for each $\lambda$ with $|\lambda|<a$. The case $a$ is not in supp $\mathfrak{a}^{\prime}$ can be treated as follows. Take $\lambda$ with $|\lambda|<a$. Since $\sup \left\{x ; x \in \operatorname{supp} \mathfrak{a}^{\prime}\right\}=a$, there are real numbers $x, y$ such that $\mid \lambda_{1}^{\prime}<x<y<a$ and $a^{\prime}((x, y))>0$. Applying again the Cauchy integral formula, the Schwarz inequality and integrating over the interval $(x, y)$ with respect to $a^{\prime}$, we have $\kappa_{s_{a^{\prime}}}(\lambda) \leqq y^{2}(x-|\lambda|)^{-2}$ $\mathfrak{a}^{\prime}((x, y))^{-1}$. All this proves (41).

Conditions (40) and (41) imply $\operatorname{disc}(0 ; a) \subset \gamma(S)$. Since support of $\mathfrak{m}$ is contained in $\operatorname{disc}(0 ; a)^{-}$, applying $[6$, Lemma 2$]$, we get

$$
\sigma_{p}\left(S^{*}\right)^{*} \subset \sigma\left(S^{-}\right) \subset \operatorname{disc}(0 ;\|S\|)^{-}=\operatorname{disc}\left(0 ;\left\|N_{\mu}\right\|\right)^{-} \subset \operatorname{disc}(0 ; a)^{-} .
$$

(with convetion: for an unbounded operator its norm is understood as $+\infty$ ). This completes the proof.

Example 3. We show that, in general, the sequences $\left\{a_{k}\right\}_{k \geqq 0}$ and $\left\{b_{k}\right\}_{k=-\infty}^{+\infty}$ representing via (34) a subnormal $S$ could not be chosen in such a way that $\left\{a_{k}\right\}_{k \geq 0}$ was a Stieltjes moment sequence.

Put $a_{n}=\left(1-(-1)^{n+1}\right)(n+1)^{-1}$, for $n \geqq 0$ and $b_{n}=(1+\mathrm{i} n)^{-1}$ for $n \in \mathbb{Z}$. Since $a_{n}=\int_{-1}^{+1} x^{n} d x,\left\{a_{n}\right\}_{n \geq 0}$ is a Hamburger moment sequence. Notice that $b_{n}=$ $\int_{0}^{2 \pi} e^{\mathrm{i} n x} e^{x}\left(e^{2 \pi}-1\right)^{-1} d x$. Thus $d \mathfrak{a}(x)=d x$ and $d \mathfrak{b}(x)=e^{x}\left(e^{2 \pi}-1\right)^{-1} d x$. It is a matter of direct calculation that $\left\{b_{k}\right\}_{k=-\infty}^{+\infty}$ satisfies (35) with $c=2 \pi\left(e^{2 \pi}-1\right)^{-1}$.

Suppose there are two sequences $\left\{\tilde{a}_{k}\right\}_{n \geqq 0}$ and $\left\{\tilde{b}_{k}\right\}_{k=-\infty}^{+\infty}$, the first of which is a Stieltjes moment sequence while the other is still a trigonometric one (with $\tilde{b}_{0}=1$ ), satisfying the following condition

$$
a_{m+n} b_{m-n}=\tilde{a}_{m+n} \tilde{b}_{m-n} .
$$

Notice that necessarily $\tilde{a}_{n}=2(n+1)^{-1}$. Indeed, it follows from (42) that $\tilde{a}_{2 n}=a_{2 n}=\int_{0}^{1} x^{2 n} d(2 x)$. Since a Hausdorff moment sequence is determined by its even terms, it must be $\tilde{a}_{n}=2(n+1)^{-1}$.

Knowing $\tilde{a}_{n}$ we are able to find out $\tilde{b}_{n}$. Namely

$$
\tilde{b}_{n}=\left[(-1)^{n} e^{\pi}-1\right]\left[1+e^{\pi}+\left(e^{\pi}-1\right)(-1)^{n}\right]\left[2\left(e^{2 \pi}-1\right)(1+\mathrm{i} n)\right]^{-1} .
$$

To prove this observe that the representing measure $\mathfrak{m}$ of $S$ is given by

$$
\mathfrak{m}(\sigma)=\int_{0}^{2 \pi} \int_{-1}^{+1} \chi_{\sigma}\left(r e^{\mathrm{i} t}\right) e^{t}\left(e^{2 \pi}-1\right)^{-1} d t d r .
$$

Consider the Borel set $\sigma_{s}=\left\{r e^{\mathrm{i} t} ; 1 / 2 \leqq r \leqq 1,0 \leqq t<s\right\}$, for $0 \leqq s<2 \pi$. The above integral formula leads us directly to 


$$
\mu\left(\sigma_{s}\right)= \begin{cases}\left(e^{s}-1\right)\left[2\left(e^{\pi}-1\right)\right]^{-1} & \text { for } s \leqq \pi \\ {\left[e^{s}\left(1+e^{-\pi}\right)+e^{2 \pi}-e^{\pi}-2\right]\left[2\left(e^{2 \pi}-1\right)\right]^{-1}} & \text { for } \pi<s>2 \pi .\end{cases}
$$

Denote by $\tilde{\mathfrak{b}}$ the representing measure of $\left\{\tilde{b}_{k}\right\}_{k=-\infty}^{+\infty}$. Since the operator $S$ is bounded, it has only one representing measure $\mathrm{m}$. By (42), mt can be described by the following formula

$$
\mathfrak{m}(\sigma)=\int_{0}^{2 \pi} \int_{0}^{1} \chi_{\sigma}\left(r e^{\mathrm{i} t}\right) d(2 r) d \tilde{\mathfrak{b}}(t) .
$$

This gives us $\mathfrak{m}\left(\sigma_{s}\right)=\tilde{\mathfrak{b}}([0, s))$. Formula (44) implies $\widetilde{\mathfrak{b}}([0, s))$ is absolutely continuous in $s$ and, consequently, $\widetilde{\mathfrak{b}}$ is absolutely continuous with respect to the Lebesgue measure on $[0,2 \pi)$. Moreover

$$
\tilde{\mathfrak{b}}(\sigma)=\int_{\sigma} \frac{d}{d s} \tilde{\mathfrak{b}}([0, s)) d s .
$$

So as to get (43) it is enough to apply (44) to the above formula.

Formula (43) implies that $\tilde{b}_{n} \neq 0$ for $n \in Z$. Since also $\tilde{a}_{n} \neq 0$, the right hand side of (42) is always different from zero. However, the left hand side vanishes for all $m, n$ such that $m+n$ is odd. This leads us to contradiction.

16. In general, it would be difficult to decide, in terms of the sequences $\left\{a_{k}\right\}_{k \geqq 0}$ and $\left\{b_{k}\right\}_{k=-\infty}^{+\infty}$, whether the operator $S$ has an analytic model. In practice, having concrete forms of these sequences one can use Corollary 11 to do this. In the case of weighted shifts all this becomes simple.

Recall that $S$ is said to be a weighted shift operator (with respect to a given orthonormal basis $\left\{e_{n}\right\}_{n \geqq 0}$ if $\mathscr{D}(S)=\operatorname{lin}\left\{e_{n}\right\}_{n \geqq 0}$ and $S e_{n} \in(\boldsymbol{C} \backslash\{0\}) e_{n+1}$ for $n \geqq 0$. Such an operator is cyclic with the cyclic vector $f_{0}=e_{0}$. The GramSchmidt procedure described in Section 10 gives us here

$$
r_{n}(z)=\left\|S^{n} e_{0}\right\|^{-1} z^{n} .
$$

Thus, by (22) and (23), we have

$$
\sigma_{p}\left(S^{*}\right)^{*}=\left\{\lambda \in C ; \sum_{n=0}^{\infty}\left\|S^{n} e_{0}\right\|^{-2}|\lambda|^{2 n}<+\infty\right\} .
$$

Denoting by ${ }_{\imath}(S)=\liminf \left\|S^{n} e_{0}\right\|^{1 / n}$, by Lemma 3 , we get

$$
\gamma(S)=\operatorname{disc}(0 ; \imath(S)) \subset \sigma_{p}\left(S^{*}\right)^{*} \subset \operatorname{disc}(0 ; \imath(S))^{-} .
$$

Moreover, $\quad \sigma_{p}\left(S^{*}\right)=\operatorname{disc}\left(0 ;{ }_{\imath}(S)\right)^{-} \quad$ when $\sum_{n=0}^{\infty}\left\|S^{n} e_{0}\right\|^{-2}|\imath(S)|^{2 n}<+\infty$. Otherwise $\sigma_{p}\left(S^{*}\right)=\operatorname{disc}\left(0 ;{ }_{\imath}(S)\right)$.

If ${ }_{\imath}(S)>0$, then, due to Corollary $11, S$ has an analytic model. In particular, the conclusion $5^{\circ}$ of Proposition 6 gives us

$$
h_{\lambda}=\sum_{n=0}^{\infty}\left\|S^{n} e_{0}\right\|^{-1} z^{* n} e_{n} .
$$


Consenquetly, the reproducing kernel $K_{\boldsymbol{r}}$ of $\mathscr{H}_{\boldsymbol{r}}$ is given by

$$
K_{r}(\lambda, \mu)=\sum_{n=0}^{\infty}\left\|S^{n} e_{0}\right\|^{-2} \lambda^{* n} \mu^{n} .
$$

All what we have said above as well as Corollary 12 enables us to decribe some parts of the spectra of $S$ and $S^{* 11}$.

Suppose now $S$ is subnormal. One can show (use the fact that the sequence $\left\{\left\|S^{n} e_{0}\right\|^{1 / n}\right\}_{n \geqq 0}$ is increasing) that ${ }_{r}(S)=\|S\|$ if $S$ is bounded and $r(S)=+\infty$ otherwise.

Consider a normal extension $N_{\mathfrak{m}}$ of $S$ (cf. Theorem 5) with the measure m given by

$$
\mathfrak{m}(\sigma)=(2 \pi)^{-1} \int_{0}^{2 \pi} \int_{0}^{+\infty} \chi_{\sigma}\left(r e^{\mathrm{i} t}\right) d \mathfrak{a}\left(r^{r}\right) d t
$$

where $\mathfrak{a}$ is given by

$$
\left\|S^{n} f_{0}\right\|^{2}=\int_{0}^{+\infty} x^{2 n} d \mathfrak{a}(x), \quad n \geqq 0 .
$$

Since the bounded case has been completely described (cf. [16]) we focus our interest on an unbounded weighted shift $S$. In this case Corollaries 12 and 13 allows us to identify the spectra and their parts as follows.

\begin{tabular}{|c|c|c|c|c|c|}
\hline & $\sigma_{p}$ & $\sigma_{a p}$ & $\sigma_{r}$ & $\sigma_{c}$ & $\sigma$ \\
\hline$S$ & $\varnothing$ & $\varnothing$ & $C$ & $\varnothing$ & $C$ \\
\hline$N$ & $\Omega$ & $M$ & $\varnothing$ & $M \backslash \Omega$ & $M$ \\
\hline$S^{*}$ & $C$ & $C$ & $\varnothing$ & $\varnothing$ & $C$ \\
\hline$N^{*}$ & $\Omega$ & $M$ & $\varnothing$ & $M \backslash \Omega$ & $M$ \\
\hline
\end{tabular}

where $N=N_{\mathfrak{m}}, M=\operatorname{supp} \mathfrak{m}$ and $\Omega=\{0\}$ if $\mathfrak{m}(\{0\})=a(\{0\})>0, \Omega=\varnothing$ otherwise.

In [19] we considered the following problem: Suppose that $S$ is a closed subnormal operator. Does there exists a normal extension $N$ of $S$ acting in $\mathcal{K}$ such that

$$
\mathscr{D}(S)=\mathscr{H} \cap \mathscr{D}(N) ?
$$

As a consequence of the fact that subnormal weighted shifts always have analytic models we have solved this question in the affirmative for such operators (cf. [19]).

The creation operator

$$
2^{-1 / 2}\left(x-\frac{d}{d x}\right)
$$

${ }^{11}$ For more detailed disscusion of the bounded case consult [16]. 
acts in $\mathcal{L}^{2}(\boldsymbol{R})$ as a weighted shift with respect to the orthonormal basis of the Hermite functions $\left[17\right.$ and 18]. In this case $\left\|S^{n} e_{0}\right\|^{2}=n !, \mathfrak{m}(d z)=\pi^{-1} \exp \left(-|z|^{2}\right) d z$, $\Omega=\varnothing$ and $M=C$.

17. Having described subnormal weighted shifts we turn our interest back to the case of an arbitrary cyclic operator, trying to compare it in some way with a weighted shift.

Proposition 12. Let $S_{\imath}$ be a cyclic operator in $\mathscr{H}_{\imath}\left(\operatorname{dim} \mathscr{H}_{2}=\infty\right)$ with the cyclic vector $f_{i 0}(i=1,2)$. Suppose that there exists a bounded linear operator $T: \mathscr{H}_{1} \rightarrow$ $\mathscr{H}_{2}$ such that $T f_{1,0}=f_{2,0}$ and $T S_{1} \subset S_{2} T$. Then

(a) $\sigma_{p}\left(S_{2}^{*}\right) \subset \sigma_{p}\left(S_{1}^{*}\right), h_{1, \lambda}=T^{*} h_{2, \lambda}, \gamma\left(S_{2}\right) \subset \gamma\left(S_{1}\right)$ and $h_{1, \lambda}=\sum_{n=0}^{\infty} r_{2, n}(\lambda) * T^{*} e_{1, n}$.

(b) If $S_{2}$ has an analytic model and $\operatorname{ker} T=\{0\}$, then $S_{1}$ has an analytic model, too.

(c) If $S_{2}$ is subnormal, then $\sigma\left(S_{2}\right) \subset \sigma\left(S_{1}\right)$.

Proof. First notice that the operator $T$ has the property $T p\left(S_{1}\right) f_{1,0}=$ $p\left(S_{2}\right) f_{2,0}$. Thus

$$
\left\langle p\left(S_{1}\right) f_{1,0}, T^{*} h_{2, \lambda}\right\rangle=\left\langle p\left(S_{2}\right) f_{2,0}, h_{2, \lambda}\right\rangle=p(\lambda), \quad p \in C[z], \quad \lambda \in \sigma_{p}\left(S_{2}^{*}\right)^{*} .
$$

This means $\sigma_{p}\left(S_{2}^{*}\right) \subset \sigma_{p}\left(S_{1}^{*}\right)$ and $h_{1, \lambda}=T^{*} h_{2, \lambda}$ (cf. Proposition 6). Consequently the conclusion (a) follows directly.

Since $S_{2}$ has a model, clolin $\left\{h_{2, \lambda} ; \lambda \in \sigma_{p}\left(S_{2}^{*}\right)^{*}\right\}=\mathscr{H}_{2}$. Thus, by (a), clolin $\left\{h_{1, \lambda} ; \lambda \in \sigma_{p}\left(S_{2}^{*}\right)^{*}\right\}=T^{*}\left(\mathscr{H}_{2}\right)^{-}$. Since $\operatorname{ker} T$ is trivial, the conclusion (b) follows.

Since $T\left(\mathscr{H}_{1}\right)$ is dense in $\mathscr{H}_{2}$ and $T S_{1}^{-} \subset S_{2}^{-} T$, the conclusion (c) follows directly from [15, Th. 3.3]

Corollary 14. Suppose that $\operatorname{dim} \mathscr{H}=\infty$. Let $S$ be a cyclic operator satisfying (34) with $\left\{a_{k}\right\}_{k \geq 0}$ being a Hamburger moment sequence and $\left\{b_{k}\right\}_{k=-\infty}^{+\infty}$ such that

(i) there is $c>0$ such that the inequality (35) holds for any finite sequence of complex numbers $\zeta_{0}, \cdots, \zeta_{k}$,

(ii) the representing measure of the trigonometric moment problem $\left\{b_{k}\right\}_{k=-\infty}^{+\infty}$ is absolutely continuous with respect to the Lebesque measure on $[0,2 \pi)$.

Then $S$ has an analytic model.

Proof. Let $\mathfrak{m}, \mathfrak{a}$ and $\mathfrak{b}$ be as in the proof of Theorem 10. In particular,

$$
\mathfrak{m}(\sigma)=\int_{0}^{2 \pi} \int_{-\infty}^{+\infty} \chi_{\sigma}\left(r e^{\mathrm{i} t}\right) d \mathfrak{a}(r) d \mathfrak{b}(t) \quad \text { for Borel } \sigma .
$$

Let $S_{a}$ be a subnormal weighted shift in $\mathscr{H}$ satisfying (34) with $b_{m}=\delta_{m}$. Then its representing measure $\mathfrak{m}_{a}$ is given by

$$
\mathfrak{m}_{a}(\sigma)=(2 \pi)^{-1} \int_{0}^{2 \pi} \int_{-\infty}^{+\infty} \chi_{\sigma}\left(r e^{i t}\right) d \mathfrak{a}(r) d t
$$


Since $\mathfrak{b}$ is absolutely continuous with respect to the Lebesque measure on $[0,2 \pi$ ), the measure $\mathfrak{m}$ is absolutely continuous with respect to $\mathfrak{m}_{a}$.

Set $T p=p$, for $p \in \mathbb{C}[z]$. Then, by (40), $T$ extends to a bounded operator from $\mathscr{H}_{\mathrm{m}}$ to $\mathscr{H}_{\mathrm{n}_{a}}$. Since the range of $T$ is dense, the only thing we have to show is, as required in Proposition 12 , that $\operatorname{ker} T$ is trivial. Take $f \in \mathscr{H}_{\mathrm{m}}$ such that $T f=0$. Then there exists a sequence of polynomials $p_{n} \in \mathbb{C}[z]$ such that $\left\{p_{n}\right\}_{n \geqq 0}$ converges to $f$ in $\mathcal{L}^{2}(\mathfrak{m})$ and to zero in $\mathcal{L}^{2}\left(\mathfrak{m}_{a}\right)$. Passing to an almost everywhere convergent subsequence we infer that $p_{n} \rightarrow f$ m-almost everywhere and $p_{n} \rightarrow 0 \mathfrak{m}_{a}$-almost everywhere. Therefore $f=0 \mathfrak{m}$-almost everywhere, which proves $\operatorname{ker} T=\{0\}$.

It is easy to check that $T S_{\mathfrak{m}} \subset S_{\mathfrak{m}_{a}} T$ and $T \mathbb{1}_{\mathfrak{m}}=\mathbb{1}_{\mathfrak{m}_{a}}$. Since $S_{\mathfrak{m}_{a}}$ has an analytic model, an application of Proposition 12(b), yields $S$ has an analytic model, too.

Remark 4. The assumptions concerning $\left\{b_{k}\right\}_{k=-\infty}^{+\infty}$ we have made in Theorem 10 can be rephrased as follows: the condition (35) is equivalent to the fact that there is a positive measure $\mathfrak{n}$ on $[0,2 \pi)$ such that $d \mathfrak{b}(t)=c(2 \pi)^{-1} d t+d \mathfrak{n}(t)$. The assumption (ii) of Corollary 14 and (35) says that $d \mathfrak{b}(t)=h(t) d t$, where $h \geqq c$ almost everywhere. If $h$ were bounded from above, then the operator $T$ of Corollary 14 would be a similarity.

\section{References}

[1] Akhiezer, N. I., Glazman, I. M., Theory of linear operators in Hilbert space, vol. 1 , Pitman, Boston-London-Melbourne, 1984.

[2] Aronszajn, N., Theory of reproducing kernels, Trans. Amer. Math. Soc., 68 (1950), 337-404.

[ 3 ] Abrahamse, M. B., Douglas, R. G., A class of subnormal operators related to multiplyconnected domains, Adv. Math., 19 (1976), 106-148.

[4] Bargmann, V., On a Hilbert space of analytic functions and associated integral transform, Comm. Pure Appl. Math., 19 (1961), 187-214.

[5] Berberian, S.K., Measure and integration, Macmillan, New York, 1965.

[6] Bram, J., Subnormal operators, Duke Math. J., 22 (1955), 75-94.

[7] Coddington, E. A., Formally normal operators having no normal extension, Can. J. Math., 17 (1965), 1030-1040

[8] Conway, J.B., Subnormal operators, Pitman, London, 1981.

[9] Frankfurt, R., Subnormal weighted shifts and related function spaces, J. Math. Anal. Appl., 52 (1970̃), 471-489.

[10] - Function spaces associated with radially symmetric measures, J. Math. Anal Appl., 60 (1977), 502-541.

[11] Fuglede, B., The multidimensional moment problem, Expo. Math., 1 (1983), 47-65.

[12] Halmos, P., Spectra and spectral manifolds, Ann. Soc. Polon. Math., 25 (1952), $43-49$.

[13] IIcDonald, G., Sundberg, C., On the spectra oi unbounded subnormal operators, Can. J. Math., 38 (1986), 1135-1148.

[14] O in, R.F., Functional relationships between a subnormal operator and its minimal 
normal extension, Pac. J. Math., 63 (1976), 221-229.

[15] Ôta, S., Schmüdgen, K., On some classes of unbounded operators, Integral Equations Operator Theory, 12 (1989), 211-226.

[16] Shields, A. L., Weighted shifts and analytic function theory, Mathematical Surveys, vol. 13, pp. 49-128, American Mathematical Society, Providence, RI, 1974.

[17] Stochel, J., Szafraniec, F.H., On normal extensions of unbounded operators. I, J. Operator Theory, 14 (1985), 31-55.

[18] - On normal extensions of unbounded operators. II, to appear in Acta Sci. Math. (Szeged), 53 (1989) ; circulating since November 1985 as Preprint No. 349 of the Institute of Mathematics, Polish Academy of Sciences.

[19] — A few assorted questions about unbounded subnormal operators, to appear in Univ. Iagel. Acta Math.

[20] Trent, T. T., $H^{2}(\mu)$ spaces and bounded point evaluation, Pac. J. Math., 80 (1979), $279-292$.

[21] — Extension of a theorem of Szegö, Michigan Math. J., 20̉ (1979), 373-377. 
